\title{
On the largest prime factor of integers
}

\author{
by \\ Chaohua Jia (Beijing) and Ming-Chit Liu (Hong Kong)
}

1. Introduction. Let $Q(x)$ denote the largest prime factor of

$$
\prod_{x<n \leq x+x^{\frac{1}{2}+\varepsilon}} n .
$$

We are interested in a lower bound of $Q(x)$. On the Riemann Hypothesis, one can show that $Q(x)>x$ holds for sufficiently large $x$.

In 1973, Jutila [11] showed that, for sufficiently large $x, Q(x)>x^{\varphi}$, where $\varphi=\frac{2}{3}-\varepsilon$. Balog [1], [2] improved it to $\varphi=0.772$. Balog, Harman and Pintz [3] obtained $\varphi=0.82$. Heath-Brown [7] got $\varphi=\frac{11}{12}-\varepsilon$. Recently, Heath-Brown and C. Jia [8] showed $\varphi=\frac{17}{18}-\varepsilon$.

In this paper, we use some ideas coming from [5], [6], and [8]-[10] on the sieve method and a delicate application of the estimate of Deshouillers and Iwaniec [4] on the mean value of Dirichlet polynomials and $\zeta$ function. Then we can prove the following:

TheOREM. Let $\varepsilon$ be a sufficiently small positive constant. Then, for sufficiently large $x$, we have

$$
Q(x)>x^{\frac{25}{26}-\varepsilon} \text {. }
$$

Throughout this paper, we suppose that $\varepsilon$ is a sufficiently small positive constant and that $B=B(\varepsilon)$ is a sufficiently large positive constant. We choose $\varepsilon$ such that $K=\frac{8}{\varepsilon}\left(\frac{1}{26}+\frac{\varepsilon}{2}\right)$ is an integer. Suppose that $x\left(>x_{0}(\varepsilon)\right)$ is sufficiently large,

$$
v=x^{\frac{25}{26}-\frac{\varepsilon}{2}}, \quad P=x^{\frac{\varepsilon}{8}}, \quad T_{0}=x^{\frac{1}{2}-\frac{\varepsilon}{6}} .
$$

Let $c, c_{1}$ and $c_{2}$ denote positive constants which have different values at different places. $m \sim M$ means that there are positive constants $c_{1}$ and $c_{2}$ such that $c_{1} M<m \leq c_{2} M$.

2000 Mathematics Subject Classification: 11N36.

Project supported partially by the Natural Science Foundation of China. 
We often use $M(s)$ ( $M$ standing here for any capital letter except $P$ and $L)$ to denote a Dirichlet polynomial in the form

$$
M(s)=\sum_{m \sim M} \frac{a(m)}{m^{s}},
$$

where $a(m)$ is a sequence of complex numbers with $a(m)=O(1)$. We also use $P(s)$ to denote

$$
P(s)=\sum_{P<p \leq 2 P} \frac{1}{p^{s}},
$$

where $p$ denotes a prime number.

All calculations in this paper can be verified on the PC computer. The paper containing full details is obtainable from the authors.

\section{Some preliminary lemmas}

Lemma 1. Suppose that $M N=v$ and that $M(s), N(s)$ are Dirichlet polynomials. Let $b=1+1 / \log x, T_{1}=\log ^{2 B} x$. Assume that $v / x^{\frac{1}{2}} \ll M \ll$ $x^{\frac{1}{2}}$. Then for $T_{1} \leq T \leq T_{0}$, we have

$$
\int_{T}^{2 T}\left|M(b+i t) N(b+i t) P^{K}(b+i t)\right| d t \ll \log ^{-B} x .
$$

This is Lemma 1 of [8].

Lemma 2. Suppose that $M N L=v$ and that $M(s), N(s)$ are Dirichlet polynomials,

$$
L(s)=\sum_{l \sim L} \frac{1}{l^{s}} .
$$

Let $b=1+1 / \log x, T_{2}=\sqrt{L}$. Assume that $M \ll v^{\frac{13}{25}}, N \ll v^{\frac{6.5}{25}}$. Then for $T_{2} \leq T \leq T_{0}$, we have

$$
\int_{T}^{2 T}\left|M(b+i t) N(b+i t) L(b+i t) P^{K}(b+i t)\right| d t \ll \log ^{-B} x .
$$

This can be proved in the same way as in Lemma 2 of [8].

Lemma 3. Suppose that $M N D L=v$ and that $M(s), N(s), D(s)$ are Dirichlet polynomials,

$$
L(s)=\sum_{l \sim L} \frac{1}{l^{s}} .
$$

Let $b=1+1 / \log x, T_{2}=\sqrt{L}$. Assume further that $M \ll v^{\frac{13}{25}}$ and that $N, D$ lie in one of the following regions: 
(i) $N \ll v^{\frac{2.6}{25}}, D \ll N^{-\frac{1}{2}} v^{\frac{6.5}{25}}$;

(ii) $v^{\frac{2.6}{25}} \ll N \ll v^{\frac{26}{175}}, D \ll N^{-\frac{4}{3}} v^{\frac{26}{75}}$;

(iii) $v^{\frac{26}{175}} \ll N \ll v^{\frac{5.2}{25}}, D \ll N^{-\frac{3}{4}} v^{\frac{6.5}{25}}$;

(iv) $v^{\frac{5.2}{25}} \ll N \ll v^{\frac{6.5}{25}}, D \ll N^{-2} v^{\frac{13}{25}}$.

Then for $T_{2} \leq T \leq T_{0}$, we have

$$
\int_{T}^{2 T}\left|M(b+i t) N(b+i t) D(b+i t) L(b+i t) P^{K}(b+i t)\right| d t \ll \log ^{-B} x .
$$

Proof. If $v / x^{\frac{1}{2}} \ll M \ll v^{\frac{13}{25}}$, it can be dealt with by Lemma 1 . We assume that $M \ll v / x^{\frac{1}{2}}$. Let $M(s) P^{K}(s)=H(s)$. Then $H=M P^{K} \ll x^{\frac{1}{2}}$. It suffices to show

$$
\begin{aligned}
I & =\int_{T}^{2 T}\left|M\left(\frac{1}{2}+i t\right) N\left(\frac{1}{2}+i t\right) D\left(\frac{1}{2}+i t\right) L\left(\frac{1}{2}+i t\right) P^{K}\left(\frac{1}{2}+i t\right)\right| d t \\
& =\int_{T}^{2 T}\left|H\left(\frac{1}{2}+i t\right) N\left(\frac{1}{2}+i t\right) D\left(\frac{1}{2}+i t\right) L\left(\frac{1}{2}+i t\right)\right| d t \\
& \ll x^{\frac{1}{2}} \log ^{-B} x .
\end{aligned}
$$

Applying Cauchy's inequality and the mean value estimate for Dirichlet polynomials, we obtain

$$
\begin{aligned}
I & \ll\left(\int_{T}^{2 T}\left|H\left(\frac{1}{2}+i t\right)\right|^{2} d t\right)^{\frac{1}{2}}\left(\int_{T}^{2 T}\left|N\left(\frac{1}{2}+i t\right) D\left(\frac{1}{2}+i t\right)\right|^{2}\left|L\left(\frac{1}{2}+i t\right)\right|^{2} d t\right)^{\frac{1}{2}} \\
& \ll x^{\frac{1}{4}}\left(\int_{T}^{2 T}\left|N\left(\frac{1}{2}+i t\right) D\left(\frac{1}{2}+i t\right)\right|^{2}\left|L\left(\frac{1}{2}+i t\right)\right|^{2} d t\right)^{\frac{1}{2}} .
\end{aligned}
$$

If $D \ll N$, an application of Theorem 2 of Deshouillers and Iwaniec [4] yields

$$
\begin{aligned}
\int_{T}^{2 T} \mid N\left(\frac{1}{2}+i t\right) D\left(\frac{1}{2}+i t\right) & ||^{2}\left|L\left(\frac{1}{2}+i t\right)\right|^{2} d t \\
& \ll T^{\frac{\varepsilon}{4}}\left(T+T^{\frac{1}{2}} N^{\frac{3}{4}} D+T^{\frac{1}{2}} N D^{\frac{1}{2}}+N^{\frac{7}{4}} D^{\frac{3}{2}}\right) .
\end{aligned}
$$

Thus, when $N, D$ lie in one of the following regions:

(a) $N \ll v^{\frac{26}{175}}, D \ll N$;

(b) $v^{\frac{26}{175}} \ll N \ll v^{\frac{5.2}{25}}, D \ll N^{-\frac{3}{4}} v^{\frac{6.5}{25}}$;

(c) $v^{\frac{5.2}{25}} \ll N \ll v^{\frac{6.5}{25}}, D \ll N^{-2} v^{\frac{13}{25}}$, 
we have

$$
\int_{T}^{2 T}\left|N\left(\frac{1}{2}+i t\right) D\left(\frac{1}{2}+i t\right)\right|^{2}\left|L\left(\frac{1}{2}+i t\right)\right|^{2} d t \ll T_{0}^{1+\frac{\varepsilon}{4}}
$$

If $D \gg N$, changing the roles of $N$ and $D$, we get

$$
\begin{aligned}
\int_{T}^{2 T} \mid D\left(\frac{1}{2}+i t\right) N\left(\frac{1}{2}+i t\right) & ||^{2}\left|L\left(\frac{1}{2}+i t\right)\right|^{2} d t \\
& \ll T^{\frac{\varepsilon}{4}}\left(T+T^{\frac{1}{2}} D^{\frac{3}{4}} N+T^{\frac{1}{2}} D N^{\frac{1}{2}}+D^{\frac{7}{4}} N^{\frac{3}{2}}\right) .
\end{aligned}
$$

Thus, when $N, D$ lie in one of the following regions:

(d) $N \ll v^{\frac{2.6}{25}}, N \ll D \ll N^{-\frac{1}{2}} v^{\frac{6.5}{25}}$;

(e) $v^{\frac{2.6}{25}} \ll N \ll v^{\frac{26}{175}}, N \ll D \ll N^{-\frac{4}{3}} v^{\frac{26}{75}}$,

we have

$$
\int_{T}^{2 T}\left|N\left(\frac{1}{2}+i t\right) D\left(\frac{1}{2}+i t\right)\right|^{2}\left|L\left(\frac{1}{2}+i t\right)\right|^{2} d t \ll T_{0}^{1+\frac{\varepsilon}{4}}
$$

Combining the regions (a)-(e), we get the regions in Lemma 3. If $N, D$ lie in one of these regions, then

$$
I \ll x^{\frac{1}{4}} T_{0}^{\frac{1}{2}+\frac{\varepsilon}{8}} \ll x^{\frac{1}{2}} \log ^{-B} x .
$$

This completes the proof of Lemma 3.

We define $w(u)$ as the continuous solution of the equations

$$
\begin{cases}w(u)=1 / u, & 1 \leq u \leq 2 \\ (u w(u))^{\prime}=w(u-1), & 2<u .\end{cases}
$$

In particular, when $2 \leq u \leq 3$,

$$
w(u)=\frac{1+\log (u-1)}{u} .
$$

Lemma 4. For the function $w(u)$, we have the following bounds:

(i) $w(u) \leq 1 / 1.763$ if $u \geq 2$;

(ii) $w(u) \leq 0.5644$ if $u \geq 3$;

(iii) $w(u) \leq 0.5617$ if $u \geq 4$.

See Lemma 5 of [8].

Define

$$
g(u)= \begin{cases}1 / u & \text { if } 1 \leq u \leq 2 \\ (1+\log (u-1)) / u & \text { if } 2<u \leq 3 \\ 0.5644 & \text { if } 3<u \leq 4 \\ 0.5617 & \text { if } 4<u\end{cases}
$$


We see that for $u \geq 1$,

$(2)$

$$
w(u) \leq g(u) .
$$

Lemma 5. Let $\mathcal{E}=\{n: t<n \leq 2 t\}$ and $z \leq t$. Set

$$
P(z)=\prod_{p<z} p, \quad S(\mathcal{E}, z)=\sum_{\substack{t<n \leq 2 t \\(n, P(z))=1}} 1 .
$$

Then for sufficiently large $t$ and $z$, we have

$$
S(\mathcal{E}, z)=w\left(\frac{\log t}{\log z}\right) \frac{t}{\log z}+O\left(\frac{t}{\log ^{2} z}\right) .
$$

See Lemma 6 of [8].

3. Sieve method. Let

$$
\begin{gathered}
N(d)=\sum_{\substack{x<d p_{1} \ldots p_{K} \leq x+x^{\frac{1}{2}+\varepsilon} \\
P<p_{i} \leq 2 P}} 1, \\
\mathcal{A}=\left\{n: 2^{-K} v<n \leq 2 v, n \text { repeats } N(n) \text { times }\right\}, \\
\mathcal{A}_{d}=\{a: a \in \mathcal{A}, d \mid a\}, \\
P(z)=\prod_{p<z} p, \quad S(\mathcal{A}, z)=\sum_{\substack{a \in \mathcal{A} \\
(a, P(z))=1}} 1 .
\end{gathered}
$$

If we prove that

$$
\Phi=\sum_{\substack{x<p p_{1} \ldots p_{K} \leq x+x^{\frac{1}{2}+\varepsilon} \\ P<p_{i} \leq 2 P \\ 2^{-K} v<p \leq 2 v}} 1>0
$$

then we obtain the assertion of the Theorem.

In the following, we set

$$
\begin{aligned}
\mathcal{B} & =\{n: v<n \leq 2 v\}, \\
X & =x^{\frac{1}{2}+\varepsilon}\left(\sum_{P<p \leq 2 P} \frac{1}{p}\right)^{K} .
\end{aligned}
$$

Buchstab's identity yields

(6) $\Phi=S\left(\mathcal{A},(2 v)^{\frac{1}{2}}\right)=S\left(\mathcal{A}, v^{\frac{1}{25}}\right)-\sum_{v^{\frac{1}{25}}<p \leq v^{\frac{12}{25}}} S\left(\mathcal{A}_{p}, p\right)-\sum_{v^{\frac{12}{25}<p \leq(2 v)^{\frac{1}{2}}}} S\left(\mathcal{A}_{p}, p\right)$.

By the discussion in Lemma 8 of [8] with the application of Lemma 1, we 
can get the asymptotic formula

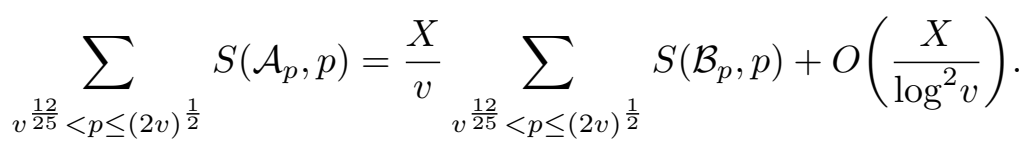

The discussion in Lemma 9 of [8] yields the asymptotic formula

$$
S\left(\mathcal{A}, v^{\frac{1}{25}}\right)=\frac{X}{v} \cdot S\left(\mathcal{B}, v^{\frac{1}{25}}\right)+O\left(\frac{X}{\log ^{2} v}\right) .
$$

Applying Buchstab's identity again, we get

$$
\begin{aligned}
& \sum_{v^{\frac{1}{25}}<p \leq v^{\frac{12}{25}}} S\left(\mathcal{A}_{p}, p\right) \\
& =\sum_{v^{\frac{1}{25}}<p \leq v^{\frac{12}{25}}} S\left(\mathcal{A}_{p}, v^{\frac{1}{25}}\right)-\sum_{v^{\frac{1}{25}}<p \leq v^{\frac{12}{25}}} \sum_{v^{\frac{1}{25}<q<p}} S\left(\mathcal{A}_{p q}, q\right) . \\
& q<\left(\frac{2 v}{p}\right)^{\frac{1}{2}}
\end{aligned}
$$

By the discussion in Lemma 9 of [8], the first sum on the right side in (9) has an asymptotic formula.

We therefore need to deal with the sum

$$
\begin{aligned}
& \sum_{v^{\frac{1}{25}<p \leq v}} \sum_{\frac{12}{25}} S\left(\mathcal{A}_{p q}, q\right) . \\
& q<\left(\frac{v}{p}\right)^{\frac{1}{2}}
\end{aligned}
$$

Removing the sum with $v^{\frac{12}{25}}<p q \leq v^{\frac{13}{25}}$ which has an asymptotic formula, we have to consider the sums

$$
\begin{aligned}
& \Omega_{1}=\sum_{v^{\frac{1}{25}}<p \leq v} \sum_{v^{\frac{11}{25}}} S\left(\mathcal{A}_{p q}, q\right), \\
& q<\frac{v \frac{12}{25}}{p}
\end{aligned}
$$

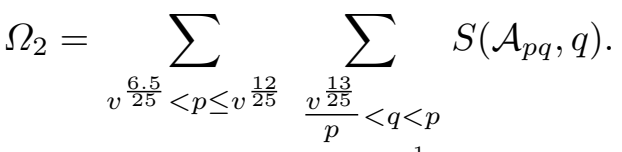

$$
\begin{aligned}
& q<\left(\frac{v}{p}\right)^{\frac{1}{2}}
\end{aligned}
$$

Now we define the deficiency of a sum as defined in Section 3 of [9]. If

$$
\Sigma=\sum_{p, q} S\left(\mathcal{A}_{p q}, q\right) \geq \frac{X}{v}(1+O(\varepsilon)) \sum_{p, q} S\left(\mathcal{B}_{p q}, q\right)-C \frac{X}{\log v},
$$

then we call the constant $C$ the deficiency of $\Sigma$. Of course any constant greater than $C$ can be used as the deficiency of $\Sigma$. If a sum has an asymptotic formula, then its deficiency is 0 . 
When we write

$$
\sum_{p, q} S\left(\mathcal{A}_{p q}, q\right) \geq 0=\frac{X}{v} \sum_{p, q} S\left(\mathcal{B}_{p q}, q\right)-\frac{X}{v} \sum_{p, q} S\left(\mathcal{B}_{p q}, q\right),
$$

by Lemma 5 and the prime number theorem, we have

$$
\begin{aligned}
\frac{X}{v} \sum_{p, q} S\left(\mathcal{B}_{p q}, q\right) & =(1+O(\varepsilon)) \frac{X}{v} \sum_{p, q} w\left(\frac{\log \frac{v}{p q}}{\log q}\right) \frac{v}{p q \log q} \\
& =(1+O(\varepsilon)) X \int \frac{d x}{x \log x} \int w\left(\frac{\log \frac{v}{x y}}{\log y}\right) \frac{d y}{y \log ^{2} y} \\
& =(1+O(\varepsilon)) \frac{X}{\log v} \int \frac{d t}{t} \int w\left(\frac{1-t-u}{u}\right) \frac{d u}{u^{2}}
\end{aligned}
$$

Hence, the deficiency is

$$
\int \frac{d t}{t} \int w\left(\frac{1-t-u}{u}\right) \frac{d u}{u^{2}}
$$

Similarly, we can define the deficiency of the sum in $2 n(n \geq 2)$ variables.

4. The deficiency of $\Omega_{1}$. Applying Buchstab's identity twice, we get

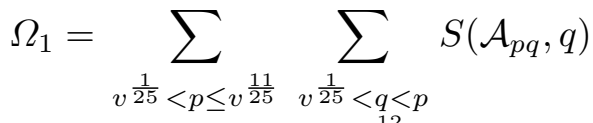

$$
\begin{aligned}
& q<\frac{v^{\frac{12}{25}}}{p} \\
& =\sum_{v^{\frac{1}{25}<p \leq v}} \sum_{v^{\frac{11}{25}}<q<p} S\left(\mathcal{A}_{p q}, v^{\frac{1}{25}}\right) \\
& q<\frac{v^{\frac{12}{25}}}{p} \\
& -\sum_{v \frac{1}{25}<p \leq v} \sum_{v^{\frac{1}{25}}<q<p} \sum_{v^{\frac{1}{25}<r<q}} S\left(\mathcal{A}_{p q r}, v^{\frac{1}{25}}\right) \\
& q<\frac{v^{\frac{12}{25}}}{p} \quad r<\left(\frac{2 v}{p q}\right)^{\frac{1}{2}} \\
& +\sum_{v^{\frac{1}{25}}<p \leq v^{\frac{11}{25}}} \sum_{v^{\frac{1}{25}<q<p}} \sum_{v^{\frac{1}{25}<r<q}} \sum_{v^{\frac{1}{25}<s<r}} S\left(\mathcal{A}_{p q r s}, s\right), \\
& q<\frac{\frac{12}{25}}{p} \quad r<\left(\frac{2 v}{p q}\right)^{\frac{1}{2}} \quad s<\left(\frac{2 v}{p q r}\right)^{\frac{1}{2}}
\end{aligned}
$$

where $p, q, r, s$ denote prime numbers.

Note that $p q<v^{\frac{13}{25}}, r<q<v^{\frac{6.5}{25}}$ in the second term in the above formula. By the discussion in Lemma 9 of [8] with the application of Lemma 2, we see that the first two terms in the above formula have asymptotic for- 
mulas. Therefore we have to deal with the sum

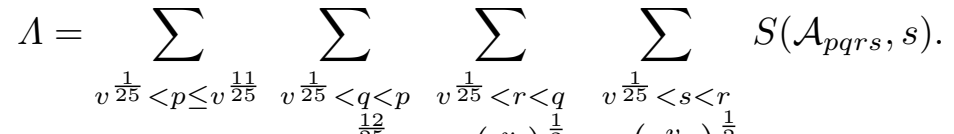

$$
\begin{aligned}
& q<\frac{v^{\frac{12}{25}}}{p} \quad r<\left(\frac{v}{p q}\right)^{\frac{1}{2}} \quad s<\left(\frac{v}{p q r}\right)^{\frac{1}{2}}
\end{aligned}
$$

We call the above procedure process $I$. In the following, we shall discuss the deficiency of $\Lambda$ in some cases. Firstly $q r s>v^{\frac{13}{25}}$ is assumed.

I. $q r s>v^{\frac{13}{25}}$. Now we discuss the deficiency of

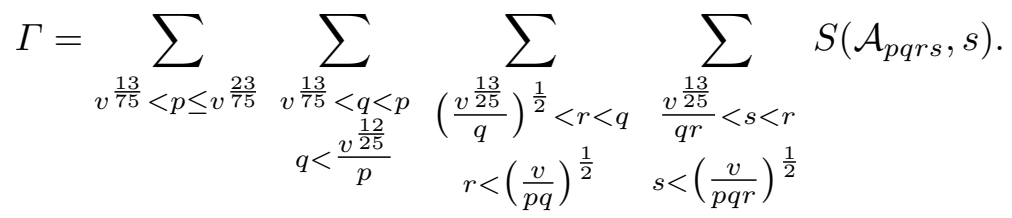

Let $\Gamma \geq 0$. Then the deficiency of $\Gamma$ is

$$
\begin{aligned}
\int_{\frac{13}{75}}^{\frac{23}{75}} \frac{d t}{t} \int_{\frac{13}{75}}^{\min \left(t, \frac{12}{25}-t\right)} \frac{d u}{u} \int_{\frac{1}{2}\left(\frac{13}{25}-u\right)}^{u} \frac{d r}{r} & \quad \int_{\frac{13}{25}-u-r}^{\min \left(r, \frac{1}{2}(1-t-u-r)\right)} w\left(\frac{1-t-u-r-s}{s}\right) \frac{d s}{s^{2}} \\
\leq & \int_{\frac{13}{75}}^{\frac{23}{75}} \int_{\frac{13}{75}}^{\frac{\min \left(t, \frac{12}{25}-t\right)}{t}} \frac{d u}{u} \int_{\frac{13}{2}\left(\frac{13}{25}-u\right)}^{u} \frac{d r}{r} \\
& \times \int_{\frac{13}{25}-u-r}^{\min \left(r, \frac{1}{2}(1-t-u-r)\right)} g\left(\frac{1-t-u-r-s}{s}\right) \frac{d s}{s^{2}} \\
\leq & 0.021170 .
\end{aligned}
$$

Next we assume $q r s<v^{\frac{13}{25}}$. The sum with $v^{\frac{12}{25}} \leq q r s<v^{\frac{13}{25}}$ can be removed, so that we can assume $q r s<v^{\frac{12}{25}}$. As in [9], we use $q r s<v^{\frac{13}{25}} \rightarrow$ $q r s<v^{\frac{12}{25}}$ to mean that the sum with $v^{\frac{12}{25}} \leq q r s<v^{\frac{13}{25}}$ is removed.

II. $q r s<v^{\frac{12}{25}}$, prs $>v^{\frac{13}{25}}$. The corresponding deficiency is

$$
\int_{\frac{5}{25}}^{\frac{11}{25}} \frac{d t}{t} \int_{\frac{1}{2}\left(\frac{13}{25}-t\right)}^{\min \left(t-\frac{1}{25}, \frac{12}{25}-t\right)} \frac{d u}{u} \int_{\frac{1}{2}\left(\frac{13}{25}-t\right)}^{u} \frac{d r}{r} \int_{\frac{13}{25}-t-r}^{\min \left(r, \frac{12}{25}-u-r\right)} w\left(\frac{1-t-u-r-s}{s}\right) \frac{d s}{s^{2}},
$$


where we used the fact that

$$
\min \left(r, \frac{12}{25}-u-r\right) \leq \frac{r}{2}+\frac{1}{2}\left(\frac{12}{25}-u-r\right)<\frac{1}{2}(1-t-u-r)
$$

We discuss several cases.

1. $r<v^{\frac{2.6}{25}}$. The corresponding deficiency is

$$
\int_{\frac{7.8}{25}}^{\frac{11}{25}} \frac{d t}{t} \int_{\frac{1}{2}\left(\frac{13}{25}-t\right)}^{\frac{12}{25}-t} \frac{d u}{u} \int_{\frac{1}{2}\left(\frac{13}{25}-t\right)}^{\min \left(u, \frac{2.6}{25}\right)} \frac{d r}{r} \int_{\frac{13}{25}-t-r}^{r} w\left(\frac{1-t-u-r-s}{s}\right) \frac{d s}{s^{2}} .
$$

Applying Buchstab's identity twice, we get

$$
\begin{aligned}
& \sum_{v^{\frac{7.8}{25}}<p \leq v^{\frac{11}{25}}} \sum_{\left(\frac{v^{\frac{13}{25}}}{p}\right)^{\frac{1}{2}}<q<\frac{v^{\frac{12}{25}}}{p}} \sum_{\left(\frac{v^{\frac{13}{25}}}{p}\right)^{\frac{1}{2}}<r<q} \sum_{\frac{v^{\frac{13}{25}}}{p r}<s<r} S\left(\mathcal{A}_{\text {pqrs }}, s\right)
\end{aligned}
$$

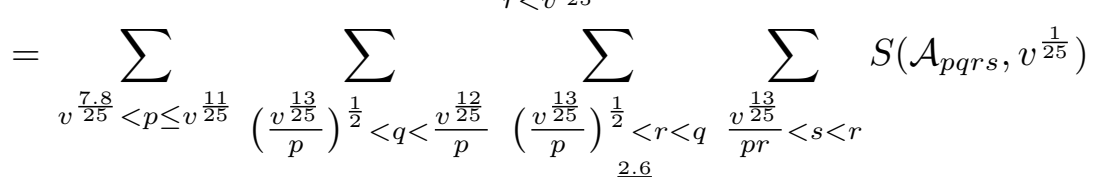

$$
\begin{aligned}
& -\sum_{v^{\frac{7.8}{25}}<p \leq v^{\frac{11}{25}}} \sum_{\left(\frac{v^{\frac{13}{25}}}{p}\right)^{\frac{1}{2}}<q<\frac{v^{\frac{12}{25}}}{p}} \sum_{\left(\frac{v^{\frac{13}{25}}}{p}\right)^{\frac{1}{2}}<r<q} \sum_{\frac{v^{\frac{13}{25}}}{p r}<s<r} \sum_{v^{\frac{1}{25}<t<s}} S\left(\mathcal{A}_{p q r s t}, v^{\frac{1}{25}}\right) \\
& r<v^{\frac{2.6}{25}} \\
& +\sum_{v^{\frac{7}{25}}<p \leq v^{\frac{11}{25}}} \sum_{\left(\frac{v^{\frac{13}{25}}}{p}\right)^{\frac{1}{2}}<q<\frac{v^{\frac{12}{25}}}{p}} \sum_{\left(\frac{v^{\frac{13}{25}}}{p}\right)^{\frac{1}{2}}<r<q} \sum_{\frac{v^{\frac{13}{25}}}{p r}<s<r} \sum_{v^{\frac{1}{25}<t<s}} \\
& r<v^{\frac{2.6}{25}} \\
& \sum_{v^{\frac{1}{25}<w<t}} S\left(\mathcal{A}_{\text {pqrstw }}, w\right)
\end{aligned}
$$

where $t$ and $w$ denote prime numbers.

In the second sum above, let $m=p q, n=r, d=s t$. Note that $s t<s^{2}<$ $r^{2}<r^{-\frac{1}{2}} v^{\frac{6.5}{25}}$. By the discussion in Lemma 9 of [8] with the application of Lemma 3 , we can get an asymptotic formula. We deal with the first sum in the same way.

The corresponding deficiency of the third sum is

$$
\begin{aligned}
\int_{\frac{7.8}{25}}^{\frac{11}{25}} \frac{d t}{t} \int_{\frac{1}{2}\left(\frac{13}{25}-t\right)}^{\frac{12}{25}-t} \frac{d u}{u} \int_{\frac{1}{2}\left(\frac{13}{25}-t\right)}^{\min \left(u, \frac{2.6}{25}\right)} \frac{d r}{r} \int_{\frac{13}{25}-t-r}^{r} \frac{d s}{s} \\
\times \int_{\frac{1}{25}}^{s} \frac{d y}{y} \int_{\frac{1}{25}}^{y} w\left(\frac{1-t-u-r-s-y-z}{z}\right) \frac{d z}{z^{2}} .
\end{aligned}
$$


1) $p t w>v^{\frac{13}{25}}$. The deficiency is

$$
\begin{aligned}
\int_{\frac{7.8}{25}}^{\frac{11}{25}} \frac{d t}{t} \int_{\frac{1}{2}\left(\frac{13}{25}-t\right)}^{\frac{12}{25}-t} \frac{d u}{u} \int_{\frac{1}{2}\left(\frac{13}{25}-t\right)}^{\min \left(u, \frac{2.6}{25}\right)} \frac{d r}{r} \int_{\frac{1}{2}\left(\frac{13}{25}-t\right)}^{r} \frac{d s}{s} \\
\times \int_{\frac{1}{2}\left(\frac{13}{25}-t\right)}^{s} \frac{d y}{y} \int_{\frac{13}{25}-t-y}^{y} g\left(\frac{1-t-u-r-s-y-z}{z}\right) \frac{d z}{z^{2}} \\
\leq 0.000108 .
\end{aligned}
$$

We now discuss the remaining cases which are similar to case 1), and write the deficiencies in brackets.

2) $\left.p t w<v^{\frac{12}{25}}, p s w>v^{\frac{13}{25}}(0.000006) .3\right) p s w<v^{\frac{12}{25}}, p r w>v^{\frac{13}{25}}$ $(0.000010)$. 4) prw $<v^{\frac{12}{25}}$, prt $>v^{\frac{13}{25}}(0.000033)$. 5) prt $<v^{\frac{12}{25}}, p q t>v^{\frac{13}{25}}$ (0.000013). 6) $p q t<v^{\frac{12}{25}}(0.000012)$.

Therefore the total deficiency in 1 . is 0.000182 .

2. $v^{\frac{2.6}{25}}<r \leq v^{\frac{26}{175}}$. The corresponding deficiency is

$$
\begin{aligned}
\int_{\frac{39}{175}}^{\frac{9.4}{25}} \frac{d t}{t} \int_{\max \left(\frac{1}{2}\left(\frac{13}{25}-t\right), \frac{2.6}{25}\right)}^{\min \left(t-\frac{1}{25}, \frac{12}{25}-t\right)} \frac{d u}{u} \int_{\frac{\max \left(\frac{1}{2}\left(\frac{13}{25}-t\right), \frac{2.6}{25}\right)}{\min \left(u, \frac{26}{175}\right)} \frac{d r}{r}} & \times \int_{\frac{13}{25}-t-r}^{\min \left(r, \frac{12}{25}-u-r\right)} w\left(\frac{1-t-u-r-s}{s}\right) \frac{d s}{s^{2}} .
\end{aligned}
$$

1) $s<r^{-\frac{2}{3}} v^{\frac{13}{75}}$. The corresponding deficiency is

$$
\begin{array}{r}
\int_{\frac{52}{175}}^{\frac{9.4}{25}} \frac{d t}{t} \int_{\max \left(\frac{2.6}{25}, \frac{26}{25}-3 t\right)}^{\frac{12}{25}-t} \frac{d u}{u} \int_{\max \left(\frac{2.6}{25}, \frac{26}{25}-3 t\right)}^{\min \left(u, \frac{26}{175}\right)} \frac{d r}{r} \\
\times \int_{\frac{13}{25}-t-r}^{\frac{13}{75}-\frac{2}{3} r} w\left(\frac{1-t-u-r-s}{s}\right) \frac{d s}{s^{2}} .
\end{array}
$$

We use the discussion in 1 . Let $m=p q, n=r, d=s t$. Note that $s t<s^{2}<$ $r^{-\frac{4}{3}} v^{\frac{26}{75}}$. We have to deal with a sum whose deficiency is

$$
\begin{aligned}
\int_{\frac{52}{175}}^{\frac{9.4}{25}} \frac{d t}{t} \int_{\max \left(\frac{2.6}{25}, \frac{26}{25}-3 t\right)}^{\frac{12}{25}-t} \frac{d u}{u} \int_{\max \left(\frac{2.6}{25}, \frac{26}{25}-3 t\right)}^{\min \left(u, \frac{26}{175}\right)} \frac{d r}{r} \int_{\frac{13}{25}-t-r}^{\frac{13}{75}-\frac{2}{3} r} \frac{d s}{s} \\
\times \int_{\frac{1}{25}}^{s} \frac{d y}{y} \int_{\frac{1}{25}}^{y} w\left(\frac{1-t-u-r-s-y-z}{z}\right) \frac{d z}{z^{2}} .
\end{aligned}
$$


We discuss the following cases.

i) $p t w>v^{\frac{13}{25}}(0.000029)$. ii) $p t w<v^{\frac{12}{25}}, p s w>v^{\frac{13}{25}}$ (0.000006). iii) $p s w<v^{\frac{12}{25}}$, prw $>v^{\frac{13}{25}}(0.000175)$. iv) prw $<v^{\frac{12}{25}}$, prt $>v^{\frac{13}{25}}(0.000041)$. v) $p r t<v^{\frac{12}{25}}, p q t>v^{\frac{13}{25}}(0.000015)$. vi) $p q t<v^{\frac{12}{25}}(0.000013)$.

Hence, the total deficiency in 1) is 0.000279 .

2) $s>r^{-\frac{2}{3}} v^{\frac{13}{75}}$. The corresponding deficiency is

$$
\begin{aligned}
\int_{\frac{39}{175}}^{\frac{9.4}{25}} \frac{d t}{t} \int_{\max \left(\frac{1}{2}\left(\frac{13}{25}-t\right), \frac{2.6}{25}\right)}^{\min \left(t-\frac{1}{25}, \frac{12}{25}-t\right)} \frac{d u}{u} \int_{\max \left(\frac{1}{2}\left(\frac{13}{25}-t\right), \frac{2.6}{25}\right)}^{\min \left(u, \frac{26}{175}\right)} \frac{d r}{r} \\
\times \int_{\max \left(\frac{13}{25}-t-r, \frac{13}{75}-\frac{2}{3} r\right)}^{\min \left(r, \frac{12}{25}-u-r\right)} w\left(\frac{1-t-u-r-s}{s}\right) \frac{d s}{s^{2}} .
\end{aligned}
$$

We have to estimate the deficiency of the sum

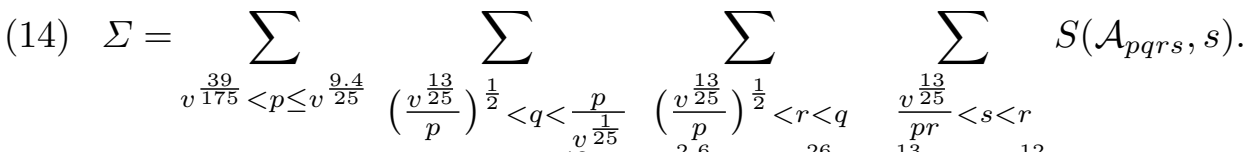

$$
\begin{aligned}
& v^{\frac{2.6}{25}}<q<\frac{v^{\frac{12}{25}}}{p} \quad v^{\frac{2.6}{25}}<r<v \frac{26}{175} \quad \frac{v \frac{13}{75}}{r^{\frac{2}{3}}}<s<\frac{v^{\frac{12}{25}}}{q r}
\end{aligned}
$$

We shall employ Buchstab's identity in the following way:

$$
\begin{aligned}
S\left(\mathcal{A}_{\text {pqrs }}, s\right)= & S\left(\mathcal{A}_{\text {pqrs }},\left(\frac{2 v}{p q r s}\right)^{\frac{1}{2}}\right) \\
& +\sum_{s \leq k<\max \left(s,\left(\frac{2 v}{p q r s}\right)^{\frac{1}{2}}\right)} S\left(\mathcal{A}_{\text {pqrsk }}, k\right),
\end{aligned}
$$

providing pqr $^{2}<v$.

The first term on the right side of (15) counts prime numbers and the second term counts almost-prime numbers. If $p q r s^{3}>2 v$, then the second term is 0 .

An application of the decomposition in (15) yields

$$
\begin{aligned}
& \Sigma=\sum_{v^{\frac{39}{175}<p \leq v^{\frac{9.4}{25}}}} \sum_{\left(\frac{v^{\frac{13}{25}}}{p}\right)^{\frac{1}{2}}<q<\frac{p}{v^{\frac{1}{25}}}} \sum_{\left(\frac{v^{\frac{13}{25}}}{p}\right)^{\frac{1}{2}}<r<q} \\
& v^{\frac{2.6}{25}}<q<\frac{v^{\frac{12}{25}}}{p} \quad v^{\frac{2.6}{25}}<r<v^{\frac{26}{175}} \\
& \sum_{\frac{13}{25}} S\left(\mathcal{A}_{p q r s},\left(\frac{2 v}{p q r s}\right)^{\frac{1}{2}}\right) \\
& \frac{v^{\frac{13}{75}}}{r^{\frac{2}{3}}}<s<\frac{v^{\frac{12}{25}}}{q r}
\end{aligned}
$$




$$
\begin{aligned}
& +\sum_{v^{\frac{39}{175}<p \leq v^{\frac{9.4}{25}}}} \sum_{\left(\frac{v^{\frac{13}{25}}}{p}\right)^{\frac{1}{2}}<q<\frac{p}{v^{\frac{1}{25}}}} \sum_{\left(\frac{v^{\frac{13}{25}}}{p}\right)^{\frac{1}{2}}<r<q} \\
& v^{\frac{2.6}{25}}<q<\frac{v^{\frac{12}{25}}}{p} \quad v^{\frac{2.6}{25}}<r<v^{\frac{26}{175}} \\
& \sum_{\frac{v^{\frac{13}{25}}}{p r}<s<r} \sum_{s \leq k<\left(\frac{2 v}{p q r s}\right)^{\frac{1}{2}}} S\left(\mathcal{A}_{\text {pqrsk }}, k\right) \\
& \frac{v^{\frac{13}{75}}}{r^{\frac{2}{3}}}<s<\frac{v^{\frac{12}{25}}}{q r} \\
& s<\left(\frac{2 v}{p q r}\right)^{\frac{1}{3}} \\
& =\Sigma_{1}+\Sigma_{2} .
\end{aligned}
$$

We call the above procedure process $I I$. We call $\Sigma_{1}$ the prime term and $\Sigma_{2}$ the almost-prime term.

i) The deficiency of the prime term is

$$
\begin{aligned}
\int_{\frac{39}{175}}^{\frac{9.4}{25}} \frac{d t}{t} \int_{\max \left(\frac{1}{2}\left(\frac{13}{25}-t\right), \frac{2.6}{25}\right)}^{\min \left(t-\frac{1}{25}, \frac{12}{25}-t\right)} \frac{d u}{u} & \int_{\max \left(\frac{1}{2}\left(\frac{13}{25}-t\right), \frac{2.6}{25}\right)}^{\min \left(u, \frac{26}{175}\right)} \frac{d r}{r} \\
\times & \int_{\max \left(\frac{13}{25}-t-r, \frac{13}{75}-\frac{2}{3} r\right)}^{\min \left(r, \frac{12}{25}-u-r\right)} \frac{d s}{s(1-t-u-r-s)} \\
\leq & 0.019316 .
\end{aligned}
$$

ii) The deficiency of the almost-prime term is

$$
\begin{aligned}
\int_{\frac{39}{175}}^{\frac{9.4}{25}} \frac{d t}{t} \int_{\max \left(\frac{1}{2}\left(\frac{13}{25}-t\right), \frac{2.6}{25}\right)}^{\min \left(t-\frac{1}{25}, \frac{12}{25}-t\right)} \frac{d u}{u} \int_{\max \left(\frac{1}{2}\left(\frac{13}{25}-t\right), \frac{2.6}{25}\right)}^{\min \left(u, \frac{26}{175}\right)} \frac{d r}{\max \left(\frac{13}{25}-t-r, \frac{13}{75}-\frac{2}{3} r\right)} \frac{d s}{s} \\
\times \int_{s}^{\frac{1}{2}(1-t-u-r-s)} w\left(\frac{1-t-u-r-s-k}{k}\right) \frac{d k}{k^{2}} .
\end{aligned}
$$

a) $k p s>v^{\frac{13}{25}}$. The corresponding deficiency is

$$
\int_{\frac{39}{175}}^{\frac{9.4}{25}} \frac{d t}{t} \int_{\max \left(\frac{1}{2}\left(\frac{13}{25}-t\right), \frac{2.6}{25}\right)}^{\min \left(t-\frac{1}{25}, \frac{12}{25}-t\right)} \frac{d u}{u} \int_{\max \left(\frac{1}{2}\left(\frac{13}{25}-t\right), \frac{2.6}{25}\right)}^{\min \left(u, \frac{26}{175}, \frac{5.5}{25}+\frac{t}{2}-u\right)} \frac{d r}{r}
$$




$$
\begin{aligned}
& \times \int_{\max \left(\frac{13}{25}-t-r, \frac{13}{75}-\frac{2}{3} r, \frac{1}{25}-t+u+r\right)}^{\min \left(r, \frac{1}{3}(1-t-u-r)\right)} \frac{d s}{s} \\
& \times \int_{\max \left(s, \frac{13}{25}-t-s\right)}^{\frac{1}{2}(1-t-u-r-s)} w\left(\frac{1-t-u-r-s-k}{k}\right) \frac{d k}{k^{2}},
\end{aligned}
$$

where we used the fact that $\frac{1}{3}(1-t-u-r) \leq \frac{12}{25}-u-r$.

$\alpha) k q r s>v^{\frac{13}{25}}(0.003549)$. $\left.\beta\right) k q r s<v^{\frac{12}{25}}(0.001012)$.

b) $k p s<v^{\frac{12}{25}}(0.000156)$.

Thus the total deficiency in 2) is 0.024033 and that in 2. is 0.024312 .

3. $r>v^{\frac{26}{175}}$. The corresponding deficiency is

$$
\begin{aligned}
\int_{\frac{5}{25}}^{\frac{58}{175}} \frac{d t}{t} \int_{\max \left(\frac{1}{2}\left(\frac{13}{25}-t\right), \frac{26}{175}\right)}^{\min \left(t-\frac{1}{25}, \frac{12}{25}-t\right)} \frac{d u}{u} \int_{\max \left(\frac{1}{2}\left(\frac{13}{25}-t\right), \frac{26}{175}\right)}^{u} \frac{d r}{r} \\
\times \int_{\frac{13}{25}-t-r}^{\min \left(r, \frac{12}{25}-u-r\right)} w\left(\frac{1-t-u-r-s}{s}\right) \frac{d s}{s^{2}} .
\end{aligned}
$$

1) The deficiency of the prime term is 0.017964 .

2) The deficiency of the almost-prime term is

$$
\begin{aligned}
\int_{\frac{5}{25}}^{\frac{58}{175}} \frac{d t}{t} \int_{\max \left(\frac{1}{2}\left(\frac{13}{25}-t\right), \frac{26}{175}\right)}^{\min \left(t-\frac{1}{25}, \frac{12}{25}-t\right)} \frac{d u}{u} \int_{\max \left(\frac{1}{2}\left(\frac{13}{25}-t\right), \frac{26}{175}\right)}^{u} \frac{d r}{r} \\
\times \int_{\frac{\min \left(r, \frac{12}{25}-u-r, \frac{1}{3}(1-t-u-r)\right)}{s}} \int_{\frac{13}{25}-t-r} \int_{s}^{\frac{1}{2}(1-t-u-r-s)} w\left(\frac{1-t-u-r-s-k}{k}\right) \frac{d k}{k^{2}} .
\end{aligned}
$$

a) $k p s>v^{\frac{13}{25}}(0.000960)$. b) $k p s<v^{\frac{12}{25}}$. There are two subcases: a) kqrs $\left.>v^{\frac{13}{25}}(0.003065) ; \beta\right) k q r s<v^{\frac{12}{25}}(0.000757)$.

Therefore the total deficiency in 3 . is 0.022746 and that in II is 0.047240 .

III. $p r s<v^{\frac{12}{25}}$, pqs $>v^{\frac{13}{25}}$. The corresponding deficiency is

$$
\int_{\frac{14}{75}}^{\frac{10}{25}} \frac{d t}{t} \int_{\frac{1}{2}\left(\frac{14}{25}-t\right)}^{\min \left(t, \frac{12}{25}-t\right)} \frac{d u}{u} \int_{\frac{13}{25}-t-u}^{u-\frac{1}{25}} \frac{d r}{r} \int_{\frac{13}{25}-t-u}^{\min \left(r, \frac{12}{25}-t-r\right)} w\left(\frac{1-t-u-r-s}{s}\right) \frac{d s}{s^{2}} \text {. }
$$


1. $q<v^{\frac{26}{175}}$. Applying process I again and writing $m=p r s, n=q$ and $d=t$, we have to deal with a sum whose deficiency is

$$
\begin{aligned}
\int_{\frac{46}{175}}^{\frac{10}{25}} \frac{d t}{t} \int_{\frac{1}{2}\left(\frac{14}{25}-t\right)}^{\min \left(\frac{12}{25}-t, \frac{26}{175}\right)} \frac{d u}{u} \int_{\frac{13}{25}-t-u}^{u-\frac{1}{25}} \frac{d r}{r} \int_{\frac{13}{25}-t-u}^{\min \left(r, \frac{12}{25}-t-r\right)} \frac{d s}{s} \\
\quad \times \int_{\frac{1}{25}}^{s} \frac{d y}{y} \int_{\frac{1}{25}}^{y} w\left(\frac{1-t-u-r-s-y-z}{z}\right) \frac{d z}{z^{2}}
\end{aligned}
$$

1) $\left.\left.p q w>v^{\frac{13}{25}}(0.000334) .2\right) p q w<v^{\frac{12}{25}}, p q t>v^{\frac{13}{25}}(0.000006) .3\right) p q t<$ $v^{\frac{12}{25}}(0.000013)$.

Hence, the total deficiency in 1 . is 0.000353 .

2. $v^{\frac{26}{175}}<q<v^{\frac{5.2}{25}}$.

1) $s<q^{-\frac{3}{4}} v^{\frac{6.5}{25}}$. Applying process I again and writing $m=$ prs, $n=q$ and $d=t$, we have to deal with a sum whose deficiency is

$$
\begin{gathered}
\int_{\frac{5.2}{25}}^{\frac{58}{175}} \frac{d t}{\max \left(\frac{1}{2}\left(\frac{14}{25}-t\right), \frac{26}{175}, \frac{26}{25}-4 t\right)} \frac{d u}{u} \int_{\frac{13}{25}-t-u}^{u-\frac{1}{25}} \frac{d r}{r} \\
\times \int_{\frac{13}{25}-t-u}^{\min \left(r, \frac{12}{25}-t-r, \frac{6.5}{25}-\frac{3}{4} u\right)} d s \frac{5.2}{25} \int_{\frac{1}{25}}^{s} \frac{d y}{y} \\
\times \min \left(y, \frac{1}{2}(1-t-u-r-s-y)\right) \\
\int_{\frac{1}{25}} w\left(\frac{1-t-u-r-s-y-z}{z}\right) \frac{d z}{z^{2}},
\end{gathered}
$$

where we used the fact that $s<\frac{12}{25}-t-r$ and $s<\frac{6.5}{25}-\frac{3}{4} u \Rightarrow t+u+r+3 s=$ $(t+r+s)+(u+2 s)<1 \Rightarrow s<\frac{1}{2}(1-t-u-r-s)$.

i) $p q w>v^{\frac{13}{25}}$ : a) pstw $>v^{\frac{13}{25}}(0.000324)$; b) pstw $<v^{\frac{12}{25}}$, prtw $>v^{\frac{13}{25}}$ (0.000108); c) prtw $<v^{\frac{12}{25}}$, prsw $>v^{\frac{13}{25}}(0.000010)$; d) prsw $<v^{\frac{12}{25}}$, prst $>$ $v^{\frac{13}{25}}(0.000006)$; e) prst $<v^{\frac{12}{25}}(0.000363)$. ii) $p q w<v^{\frac{12}{25}}, p q t>v^{\frac{13}{25}}(0.000300)$. iii) $p q t<v^{\frac{12}{25}}, p q t w>v^{\frac{13}{25}}(0.000322)$. iv) $p q t w<v^{\frac{12}{25}}(0.000006)$.

Hence, the total deficiency in 1 ) is 0.001439 .

2) $s>q^{-\frac{3}{4}} v^{\frac{6.5}{25}}(0.002038)$.

Thus the total deficiency in 2 . is 0.003477 .

3. $v^{\frac{5.2}{25}}<q\left(<v^{\frac{6.5}{25}}\right)$.

1) $s<q^{-2} v^{\frac{13}{25}}$. Applying process I again and writing $m=p r s, n=q$ and $d=t$, we have to deal with a sum whose deficiency is 


$$
\begin{aligned}
\int_{\frac{5.2}{25}}^{\frac{6.8}{25}} \frac{d t}{t} \int_{\frac{5.2}{25}}^{\min \left(t, \frac{12}{25}-t\right)} \frac{d u}{u} \int_{\frac{13}{25}-t-u}^{u-\frac{1}{25}} \frac{d r}{r} \\
\times \int_{\frac{1}{25}}^{\min \left(r, \frac{12}{25}-t-r, \frac{13}{25}-2 u\right)} \frac{d s}{s} \int_{\frac{1}{25}}^{s} \frac{d y}{y} \\
\times \int_{\frac{13}{25}-t-u}^{\min \left(y, \frac{1}{2}(1-t-u-r-s-y)\right)} w\left(\frac{1-t-u-r-s-y-z}{z}\right) \frac{d z}{z^{2}} .
\end{aligned}
$$

i) $p q w>v^{\frac{13}{25}}(0.001662)$. ii) $p q w<v^{\frac{12}{25}}, p q t>v^{\frac{13}{25}}(0.000020)$. iii) $p q t<v^{\frac{12}{25}}, p q t w>v^{\frac{13}{25}}(0.000019)$. iv) $p q t w<v^{\frac{12}{25}}(0)$.

Therefore the total deficiency in 1) is 0.001701 .

2) $s>q^{-2} v^{\frac{13}{25}}$.

i) The deficiency of the prime term is 0.009678 .

ii) The deficiency of the almost-prime term is

$$
\begin{aligned}
\int_{\frac{5.2}{25}}^{\frac{6.8}{25}} \frac{d t}{t} \int_{\frac{5.2}{25}}^{\min \left(t, \frac{12}{25}-t\right)} \frac{d u}{u} \int_{\frac{13}{25}-2 u}^{2 u-t-\frac{1}{25}} \frac{d r}{r} \int_{\frac{13}{25}-2 u}^{\min \left(r, \frac{12}{25}-t-r\right)} \frac{d s}{s} \\
\times \int_{s}^{\frac{1}{2}(1-t-u-r-s)} w\left(\frac{1-t-u-r-s-k}{k}\right) \frac{d k}{k^{2}},
\end{aligned}
$$

where we used the fact that $\min \left(r, \frac{12}{25}-t-r\right) \leq \frac{r}{3}+\frac{2}{3}\left(\frac{12}{25}-t-r\right)<$ $\frac{1}{3}(1-t-u-r)$.

a) $k p s>v^{\frac{13}{25}}(0.000006)$. b) $k p s<v^{\frac{12}{25}}, k p r>v^{\frac{13}{25}}(0.000653)$. c) $k p r<$ $\left.\left.v^{\frac{12}{25}}: \alpha\right) k q r s>v^{\frac{13}{25}}(0.002882) ; \beta\right) k q r s<v^{\frac{12}{25}}(0.001823)$.

Hence, the total deficiency in 2) is 0.015042 and that in 3 . is 0.016743 . Therefore the total deficiency in III is 0.020573 .

IV. $p q s<v^{\frac{12}{25}}, p q r>v^{\frac{13}{25}}$. The corresponding deficiency is

$$
\begin{aligned}
\int_{\frac{13}{75}}^{\frac{9}{25}} \frac{d t}{t} \int_{\frac{1}{2}\left(\frac{13}{25}-t\right)}^{\min \left(t, \frac{11}{25}-t\right)} \frac{d u}{u} \int_{\frac{13}{25}-t-u}^{u} \frac{d r}{r} \\
\quad \times \int_{\frac{1}{25}}^{\frac{12}{25}-t-u} w\left(\frac{1-t-u-r-s}{s}\right) \frac{d s}{s^{2}} .
\end{aligned}
$$

1. $r<v^{\frac{26}{175}}$. Applying process I again and writing $m=p q s, n=r$ and $d=t$, we have to deal with a sum whose deficiency is 


$$
\begin{aligned}
\int_{\frac{32.5}{175}}^{\frac{9}{25}} \frac{d t}{t} & \int_{\max \left(\frac{1}{2}\left(\frac{13}{25}-t\right), \frac{65}{175}-t\right)}^{\min \left(t, \frac{11}{25}-t\right)} \frac{d u}{u} \int_{\frac{13}{25}-t-u}^{\min \left(u, \frac{26}{175}\right)} \frac{d r}{r} \int_{\frac{1}{25}}^{\frac{12}{25}-t-u} \frac{d s}{s} \\
& \times \int_{\frac{1}{25}}^{s} \frac{d y}{y} \int_{\frac{1}{25}}^{\min \left(y, \frac{1}{2}(1-t-u-r-s-y)\right)} w\left(\frac{1-t-u-r-s-y-z}{z}\right) \frac{d z}{z^{2}} .
\end{aligned}
$$

1) prtw $>v^{\frac{13}{25}}(0.000164)$. 2) prtw $<v^{\frac{12}{25}}$, prsw $>v^{\frac{13}{25}}(0.000008)$. 3) prsw $<v^{\frac{12}{25}}$, prst $>v^{\frac{13}{25}}(0.000009)$. 4) prst $<v^{\frac{12}{25}}$, prstw $>v^{\frac{13}{25}}$ (0.000519). 5) prstw $<v^{\frac{12}{25}}(0.000269)$.

Hence, the total deficiency in 1 . is 0.000969 .

2. $v^{\frac{26}{175}}<r<v^{\frac{5.2}{25}}$.

1) $s<r^{-\frac{3}{4}} v^{\frac{6.5}{25}}$. Applying process I again and writing $m=p q s, n=r$ and $d=t$, we have to deal with a sum whose deficiency is

$$
\begin{aligned}
\int_{\frac{13}{75}}^{\frac{51}{175}} \frac{d t}{t} \int_{\max \left(\frac{1}{2}\left(\frac{13}{25}-t\right), \frac{26}{175}\right)}^{\min \left(t, \frac{11}{25}-t\right)} \frac{d u}{u} \int_{\max \left(\frac{13}{25}-t-u, \frac{26}{175}\right)}^{\min \left(u, \frac{5.2}{25}\right)} \frac{d r}{r} \\
\times \int_{\frac{1}{25}}^{\min \left(\frac{12}{25}-t-u, \frac{6.5}{25}-\frac{3}{4} r\right)} \frac{d s}{s} \int_{\frac{1}{25}}^{s} \frac{d y}{y} \\
\times \int_{\frac{1}{25}}^{\min \left(y, \frac{1}{2}(1-t-u-r-s-y)\right)} w\left(\frac{1-t-u-r-s-y-z}{z}\right) \frac{d z}{z^{2}} .
\end{aligned}
$$

i) $p r t w>v^{\frac{13}{25}}(0.000387)$. ii) prtw $<v^{\frac{12}{25}}$, prsw $>v^{\frac{13}{25}}(0.000052)$. iii) $p r s w<v^{\frac{12}{25}}$, prst $>v^{\frac{13}{25}}(0.000009)$. iv) prst $<v^{\frac{12}{25}}$, prstw $>v^{\frac{13}{25}}$ (0.000293). v) prstw $<v^{\frac{12}{25}}(0.000015)$.

Hence, the total deficiency in 1) is 0.000756 .

2) $s>r^{-\frac{3}{4}} v^{\frac{6.5}{25}}(0.000006)$.

Thus the total deficiency in 2. is 0.000762 .

3. $r>v^{\frac{5.2}{25}}(0.000108)$.

Therefore the total deficiency in IV is 0.001839 .

V. $p q r<v^{\frac{12}{25}}, p q r s>v^{\frac{13}{25}}$. The corresponding deficiency is

$$
\int_{\frac{13}{100}}^{\frac{10}{25}} \frac{d t}{t} \int_{\frac{1}{3}\left(\frac{13}{25}-t\right)}^{\min \left(t, \frac{11}{25}-t\right)} \frac{d u}{u} \int_{\frac{1}{2}\left(\frac{13}{25}-t-u\right)}^{\min \left(u, \frac{12}{25}-t-u\right)} \frac{d r}{r} \int_{\frac{13}{25}-t-u-r}^{r} w\left(\frac{1-t-u-r-s}{s}\right) \frac{d s}{s^{2}} .
$$


1. $s<v^{\frac{26}{175}}$. Applying process I again and writing $m=p q r, n=s$ and $d=t$, we have to deal with a sum whose deficiency is

$$
\begin{aligned}
& \int_{\frac{13}{100}}^{\frac{10}{25}} \frac{d t}{t} \int_{\frac{1}{3}\left(\frac{13}{25}-t\right)}^{\min \left(t, \frac{11}{25}-t\right)} \frac{d u}{u} \int_{\frac{1}{2}\left(\frac{13}{25}-t-u\right)}^{\min \left(u, \frac{12}{25}-t-u\right)} \frac{d r}{r} \\
& \times \int_{\frac{13}{25}-t-u-r}^{\min \left(r, \frac{26}{175}\right)} \frac{d s}{s} \int_{\frac{1}{25}}^{s} \frac{d y}{y} \int_{\frac{1}{25}}^{\min \left(y, \frac{1}{2}(1-t-u-r-s-y)\right)} w\left(\frac{1-t-u-r-s-y-z}{z}\right) \frac{d z}{z^{2}} .
\end{aligned}
$$

1) $\left.p r t w>v^{\frac{13}{25}}(0.000700) .2\right)$ prtw $<v^{\frac{12}{25}}, p q t w>v^{\frac{13}{25}}(0.000849)$. 3) $p q t w<v^{\frac{12}{25}}, p q s w>v^{\frac{13}{25}}(0.000515)$. 4) pqsw $<v^{\frac{12}{25}}$, pqst $>v^{\frac{13}{25}}$ : i) $p q r w>v^{\frac{13}{25}}(0.000017)$; ii) pqrw $<v^{\frac{12}{25}}(0.000374)$. 5) pqst $<v^{\frac{12}{25}}$, pqrt $>$ $v^{\frac{13}{25}}:$ i) qrstw $>v^{\frac{13}{25}}(0.000065)$; ii) qrstw $<v^{\frac{12}{25}}$, prstw $>v^{\frac{13}{25}}(0.000148)$; iii) prstw $<v^{\frac{12}{25}}(0.000910)$. 6) pqrt $<v^{\frac{12}{25}}:$ i) pqstw $>v^{\frac{13}{25}}(0.000193)$;

ii) $p q s t w<v^{\frac{12}{25}}$, pqrtw $>v^{\frac{13}{25}}(0.000008)$; iii) pqrtw $<v^{\frac{12}{25}}(0.000006)$.

Thus the total deficiency in 1 . is 0.003785 .

2. $s>v^{\frac{26}{175}}(0.000027)$.

Therefore the total deficiency in $\mathrm{V}$ is 0.003812 .

VI. $p q r s<v^{\frac{12}{25}}$. The corresponding deficiency is

$$
\begin{aligned}
\int_{\frac{1}{25}}^{\frac{9}{25}} \frac{d t}{t} \int_{\frac{1}{25}}^{\min \left(t, \frac{10}{25}-t\right)} \frac{d u}{u} \int_{\frac{1}{25}}^{\min \left(u, \frac{11}{25}-t-u\right)} \frac{d r}{r} & \times \int_{\frac{1}{25}}^{\min \left(r, \frac{12}{25}-t-u-r\right)} w\left(\frac{1-t-u-r-s}{s}\right) \frac{d s}{s^{2}} .
\end{aligned}
$$

Applying process I again, we have to deal with a sum whose deficiency is

$$
\begin{aligned}
\int_{\frac{1}{25}}^{\frac{9}{25}} \frac{d t}{t} \int_{\frac{1}{25}}^{\min \left(t, \frac{10}{25}-t\right)} \frac{d u}{u} \int_{\frac{1}{25}}^{\min \left(u, \frac{11}{25}-t-u\right)} \frac{d r}{r} \\
\times \int_{\frac{1}{25}}^{\min \left(r, \frac{12}{25}-t-u-r\right)} \frac{d s}{s} \int_{\frac{1}{25}}^{s} \frac{d y}{y} \int_{\frac{1}{25}}^{y} w\left(\frac{1-t-u-r-s-y-z}{z}\right) \frac{d z}{z^{2}},
\end{aligned}
$$

where we used the fact that $s \leq \min \left(r, \frac{12}{25}-t-u-r\right) \leq \frac{r}{2}+\frac{1}{2}\left(\frac{12}{25}-t-u-r\right)=$ $\frac{1}{2}\left(\frac{12}{25}-t-u\right)<\frac{1}{4}(1-t-u-r) \Rightarrow y<\frac{1}{2}(1-t-u-r-s-y)$.

1. prstw $>v^{\frac{13}{25}}(0.000453)$. 2. prstw $<v^{\frac{12}{25}}$, pqstw $>v^{\frac{13}{25}}(0.000324)$. 3. pqstw $<v^{\frac{12}{25}}$, pqrtw $>v^{\frac{13}{25}}(0.000303)$. 4. pqrtw $<v^{\frac{12}{25}}$, pqrsw $>v^{\frac{13}{25}}$ (0.000153). 5. pqrsw $<v^{\frac{12}{25}}$, pqrst $>v^{\frac{13}{25}}(0.000046)$. 
6. pqrst $<v^{\frac{12}{25}}$, pqrstw $>v^{\frac{13}{25}}$. Applying process I once more, we have the deficiency

$$
\begin{aligned}
& \int_{\frac{13}{150}}^{\frac{8}{25}} \frac{d t}{t} \int_{\frac{1}{5}\left(\frac{13}{25}-t\right)}^{\min \left(t, \frac{9}{25}-t\right)} \frac{d u}{u} \int_{\frac{1}{4}\left(\frac{13}{25}-t-u\right)}^{\min \left(u, \frac{10}{25}-t-u\right)} \frac{d r}{r} \\
& \times \int_{\frac{1}{3}\left(\frac{13}{25}-t-u-r\right)}^{\min \left(r, \frac{11}{25}-t-u-r\right)} \frac{d s}{s} \int_{\frac{1}{2}\left(\frac{13}{25}-t-u-r-s\right)}^{\min \left(s, \frac{12}{25}-t-u-r-s\right)} \frac{d y}{y} \int_{\frac{13}{25}-t-u-r-s-y}^{y} \frac{d z}{z} \int_{\frac{1}{25}}^{z} \frac{d \alpha}{\alpha} \\
& \times \int_{\frac{1}{25}}^{\alpha} w\left(\frac{1-t-u-r-s-y-z-\alpha-\beta}{\beta}\right) \frac{d \beta}{\beta^{2}} \\
& \leq \frac{1}{1.763} \int_{\frac{13}{150}}^{\frac{8}{25}} \frac{d t}{t} \int_{\frac{1}{5}\left(\frac{13}{25}-t\right)}^{\min \left(t, \frac{9}{25}-t\right)} \frac{d u}{u} \int_{\frac{1}{4}\left(\frac{13}{25}-t-u\right)}^{\min \left(u, \frac{10}{25}-t-u\right)} \frac{d r}{r} \\
& \times \int_{\frac{1}{3}\left(\frac{13}{25}-t-u-r\right)}^{\min \left(r, \frac{11}{25}-t-u-r\right)} \frac{d s}{s} \int_{\frac{1}{2}\left(\frac{13}{25}-t-u-r-s\right)}^{\min \left(s, \frac{12}{25}-t-u-r-s\right)} \frac{d y}{y} \\
& \times \int_{\frac{13}{25}-t-u-r-s-y}^{y}\left(25 \log (25 z)-25+\frac{1}{z}\right) \frac{d z}{z} \\
& \leq 0.000359 \text {, }
\end{aligned}
$$

where we used the fact that $\beta \leq \frac{1}{3}(1-t-u-r-s-y-z-\alpha)$ and Lemma 4 .

7. pqrstw $<v^{\frac{12}{25}}$. Applying process I twice, we have the deficiency

$$
\begin{aligned}
0.5617 \int_{\frac{1}{25}}^{\frac{7}{25}} \frac{d t}{t} \int_{\frac{1}{25}}^{\min \left(t, \frac{8}{25}-t\right)} \frac{d u}{u} \int_{\frac{1}{25}}^{\min \left(u, \frac{9}{25}-t-u\right)} \frac{d r}{r} \\
\quad \times \int_{\frac{1}{25}}^{\min \left(r, \frac{10}{25}-t-u-r\right)} \frac{d s}{s} \int_{\frac{1}{25}}^{\min \left(s, \frac{11}{25}-t-u-r-s\right)} \frac{d y}{y} \\
\times \\
\leq 0.000495,
\end{aligned}
$$

where we used the fact that $\beta \leq \frac{1}{5}(1-t-u-r-s-y-z-\alpha)$ and Lemma 4 .

Therefore the total deficiency in VI is 0.002133 . Summing up the above estimates, we conclude that the total deficiency of $\Omega_{1}$ is 0.096767 . 
5. The deficiency of $\Omega_{2}$. Now we consider the sum

$$
\Omega_{2}=\sum_{\substack{\frac{6.5}{25}<p \leq v^{\frac{12}{25}} \\ \frac{v^{\frac{13}{25}}}{p}<q<p \\ q<\left(\frac{v}{p}\right)^{\frac{1}{2}}}} S\left(\mathcal{A}_{p q}, q\right) .
$$

The corresponding deficiency is

$$
\int_{\frac{6}{25}}^{\frac{12}{25}} \frac{d t}{t} \int_{\frac{13}{25}-t}^{\min \left(t, \frac{1}{2}(1-t)\right)} w\left(\frac{1-t-u}{u}\right) \frac{d u}{u^{2}} .
$$

We discuss several cases.

I. $q<v^{\frac{2.6}{25}}$. The corresponding deficiency is

$$
\int_{\frac{10.4}{25}}^{\frac{12}{25}} \frac{d t}{t} \int_{\frac{13}{25}-t}^{\frac{2.6}{25}} w\left(\frac{1-t-u}{u}\right) \frac{d u}{u^{2}} .
$$

Let $m=p, n=q$ and $d=r$. Applying process I with Lemma 3, we have to deal with a sum whose deficiency is

$$
\int_{\frac{10.4}{25}}^{\frac{12}{25}} \frac{d t}{t} \int_{\frac{13}{25}-t}^{\frac{2.6}{25}} \frac{d u}{u} \int_{\frac{1}{25}}^{u} \frac{d r}{r} \int_{\frac{1}{25}}^{r} w\left(\frac{1-t-u-r-s}{s}\right) \frac{d s}{s^{2}} .
$$

1. $p s>v^{\frac{13}{25}}$.

1) $s<q^{-\frac{1}{4}} r^{-\frac{1}{2}} v^{\frac{6.5}{50}}$. Applying process I again and writing $m=p, n=q$ and $d=r s t$, we have to deal with a sum whose deficiency is

$$
\begin{aligned}
\int_{\frac{78}{175}}^{\frac{12}{25}} \frac{d t}{t} \int_{\frac{13}{25}-t}^{\min \left(\frac{2.6}{25}, 6 t-\frac{65}{25}\right)} \frac{d u}{u} \int_{\frac{13}{25}-t}^{\min \left(u, 2 t-\frac{u}{2}-\frac{19.5}{25}\right)} \frac{d r}{r} \int_{\frac{13}{25}-t}^{\min \left(r, \frac{6.5}{50}-\frac{u}{4}-\frac{r}{2}\right)} \frac{d s}{s} \\
\times \int_{\frac{1}{25}}^{s} \frac{d y}{y} \int_{\frac{1}{25}}^{\min \left(y, \frac{1}{2}(1-t-u-r-s-y)\right)} w\left(\frac{1-t-u-r-s-y-z}{z}\right) \frac{d z}{z^{2}} .
\end{aligned}
$$

i) $p w>v^{\frac{13}{25}}(0.000508)$. ii) $p w<v^{\frac{12}{25}}(0)$.

2) $s>q^{-\frac{1}{4}} r^{-\frac{1}{2}} v^{\frac{6.5}{50}}(0.007963)$.

Thus the total deficiency in 1 . is 0.008471 .

2. $p s<v^{\frac{12}{25}}, p r>v^{\frac{13}{25}}(0.000996)$. 3. $p r<v^{\frac{12}{25}}, p r s>v^{\frac{13}{25}}(0.000831)$. 4. $p r s<v^{\frac{12}{25}}(0)$.

Therefore the total deficiency in I is 0.010298 . 
II. $v^{\frac{2.6}{25}}<q<v^{\frac{26}{175}}$. The corresponding deficiency is

$$
\int_{\frac{65}{175}}^{\frac{12}{25}} \frac{d t}{t} \int_{\max \left(\frac{13}{25}-t, \frac{2.6}{25}\right)}^{\frac{26}{175}} w\left(\frac{1-t-u}{u}\right) \frac{d u}{u^{2}} .
$$

Let $m=p, n=q$ and $d=r$. Applying process I with Lemma 3, we have to deal with a sum whose deficiency is

$$
\int_{\frac{65}{175}}^{\frac{12}{25}} \frac{d t}{t} \int_{\max \left(\frac{13}{25}-t, \frac{2.6}{25}\right)}^{\frac{26}{175}} \frac{d u}{u} \int_{\frac{1}{25}}^{u} \frac{d r}{r} \int_{\frac{1}{25}}^{\min \left(r, \frac{1}{2}(1-t-u-r)\right)} w\left(\frac{1-t-u-r-s}{s}\right) \frac{d s}{s^{2}} .
$$

1. $p s>v^{\frac{13}{25}}$.

1) $s<q^{-\frac{2}{3}} r^{-\frac{1}{2}} v^{\frac{13}{75}}$. Let $m=p, n=q$ and $d=r s t$. Applying process I with Lemma 3, we have to deal with a sum the deficiency of which is

$$
\begin{aligned}
\int_{\frac{33.8}{75}}^{\frac{12}{25}} \frac{d t}{t} \int_{\frac{2.6}{25}}^{\min \left(\frac{26}{175}, \frac{9}{4} t-\frac{91}{100}\right)} \frac{d u}{u} \int_{\frac{13}{25}-t}^{\min \left(u, 2 t-\frac{4}{3} u-\frac{52}{75}\right)} \frac{d r}{r} \\
\times \int_{\frac{1}{25}}^{\min \left(r, \frac{13}{75}-\frac{2}{3} u-\frac{r}{2}\right)} \frac{d s}{s} \int_{\frac{1}{25}}^{s} \frac{d y}{y} \\
\times \int_{\frac{13}{25}-t}^{\min \left(y, \frac{1}{2}(1-t-u-r-s-y)\right)} g\left(\frac{1-t-u-r-s-y-z}{z}\right) \frac{d z}{z^{2}}
\end{aligned}
$$$$
\leq 0.000486 \text {. }
$$

2) $s>q^{-\frac{2}{3}} r^{-\frac{1}{2}} v^{\frac{13}{75}}(0.071032)$.

Hence, the total deficiency in 1 . is 0.071518 .

2. $p s<v^{\frac{12}{25}}, p r>v^{\frac{13}{25}}$.

1) $s<q^{-\frac{4}{3}} r^{-1} v^{\frac{26}{75}}$. Let $m=p s, n=q$ and $d=r t$. Applying process I with Lemma 3, we have to deal with a sum whose deficiency is

$$
\begin{aligned}
\int_{\frac{68}{175}}^{\frac{11}{25}} \frac{d t}{t} \int_{\max \left(\frac{13}{25}-t, \frac{2.6}{25}\right)}^{\min \left(\frac{26}{175}, \frac{3}{4} t-\frac{4}{25}\right)} \frac{d u}{u} \int_{\frac{13}{25}-t}^{\min \left(u, \frac{23}{75}-\frac{4}{3} u\right)} \frac{d r}{r} \\
\times \int_{\frac{1}{25}}^{\min \left(\frac{12}{25}-t, \frac{26}{75}-\frac{4}{3} u-r\right)} \frac{d s}{s} \int_{\frac{1}{25}}^{\min \left(s, \frac{1}{2}(1-t-u-r-s)\right)} \frac{d y}{y}
\end{aligned}
$$




$$
\times \int_{\frac{1}{25}}^{\min \left(y, \frac{1}{2}(1-t-u-r-s-y)\right)} g\left(\frac{1-t-u-r-s-y-z}{z}\right) \frac{d z}{z^{2}}
$$

$\leq 0.000290$.

2) $s>q^{-\frac{4}{3}} r^{-1} v^{\frac{26}{75}}$.

i) The deficiency of the prime term is 0.005291 .

ii) The deficiency of the almost-prime term is

$$
\begin{aligned}
\int_{\frac{65}{175}}^{\frac{11}{25}} \frac{d t}{t} \int_{\max \left(\frac{13}{25}-t, \frac{3}{7}\left(t-\frac{10}{75}\right)\right)}^{\frac{26}{175}} \frac{d u}{u} \int_{\max \left(\frac{13}{25}-t, t-\frac{4}{3} u-\frac{10}{75}\right)}^{u} \frac{d r}{r} \\
\times \int_{\frac{\max \left(\frac{1}{25}, \frac{26}{75}-\frac{4}{3} u-r\right)}{25}-t} \frac{d s}{s} \\
\times \int_{s}^{\frac{1}{2}(1-t-u-r-s)} w\left(\frac{1-t-u-r-s-k}{k}\right) \frac{d k}{k^{2}} .
\end{aligned}
$$

a) $k p>v^{\frac{13}{25}}(0.001783)$. b) $k p<v^{\frac{12}{25}}, k p s>v^{\frac{13}{25}}(0.001370)$. c) $k p s<v^{\frac{12}{25}}$ (0.000068).

Thus the total deficiency in 2 . is 0.008802 .

3. $p r<v^{\frac{12}{25}}, p r s>v^{\frac{13}{25}}$.

1) $s<q^{-\frac{2}{3}} v^{\frac{13}{75}}$. Let $m=p r, n=q$ and $d=s t$. Applying process I with Lemma 3 , we have to deal with a sum whose deficiency is

$$
\begin{aligned}
\int_{\frac{65}{175}}^{\frac{11}{25}} \frac{d t}{t} \int_{\max \left(\frac{13}{25}-t, \frac{2.6}{25}\right)}^{\frac{26}{175}} \frac{d u}{u} \int_{\frac{1}{2}\left(\frac{13}{25}-t\right)}^{\frac{12}{25}-t} \frac{d r}{r} \int_{\frac{13}{25}-t-r}^{\min \left(r, \frac{13}{75}-\frac{2}{3} u\right)} \frac{d s}{s} \\
\quad \times \int_{\frac{1}{25}}^{s} \frac{d y}{y} \int_{\frac{1}{25}}^{y} w\left(\frac{1-t-u-r-s-y-z}{z}\right) \frac{d z}{z^{2}} .
\end{aligned}
$$

i) $p t w>v^{\frac{13}{25}}(0.000088)$. ii) $p t w<v^{\frac{12}{25}}, p s w>v^{\frac{13}{25}}(0.000006)$. iii) $p s w<v^{\frac{12}{25}}$, $p r w>v^{\frac{13}{25}}(0.000011)$. iv) $p r w<v^{\frac{12}{25}}(0)$.

2) $s>q^{-\frac{2}{3}} v^{\frac{13}{75}}(0.000571)$.

Therefore the total deficiency in 3. is 0.000676 and that in II is 0.080996 .

III. $v^{\frac{26}{175}}<q<v^{\frac{5 \cdot 2}{25}}$. Now we consider the deficiency of the sum

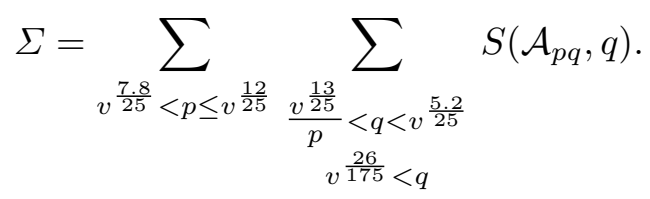


We write

$$
\Sigma=\Psi_{1}+\Psi_{2},
$$

where

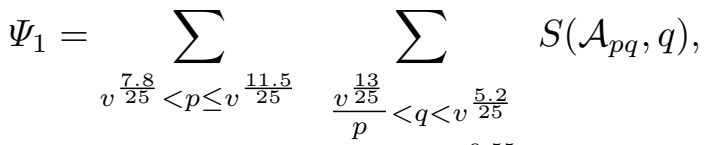

$$
\begin{aligned}
& v^{\frac{26}{175}}<q<\frac{v^{\frac{9.55}{25}}}{p^{\frac{1}{2}}} \\
& \Psi_{2}=\sum_{v^{\frac{8.7}{25}}<p \leq v^{\frac{11.5}{25}}} \sum_{\frac{v^{\frac{9.55}{25}}}{p^{\frac{1}{2}}}<q<v^{\frac{5.2}{25}}} S\left(\mathcal{A}_{p q}, q\right)+\sum_{v^{\frac{11.5}{25}<p \leq v^{\frac{12}{25}}}} \sum_{v v^{\frac{26}{175}<q<v^{\frac{5}{25}}}} S\left(\mathcal{A}_{p q}, q\right) .
\end{aligned}
$$

The deficiency of $\Psi_{2}$ is

$$
\int_{\frac{8.7}{25}}^{\frac{11.5}{25}} \frac{d t}{t} \int_{\frac{9.55}{25}-\frac{t}{2}}^{\frac{5.2}{25}} g\left(\frac{1-t-u}{u}\right) \frac{d u}{u^{2}}+\int_{\frac{11.5}{25}}^{\frac{12}{25}} \frac{d t}{t} \int_{\frac{26}{175}}^{\frac{5.2}{25}} g\left(\frac{1-t-u}{u}\right) \frac{d u}{u^{2}} \leq 0.157504 .
$$

Next we shall discuss the deficiency of $\Psi_{1}$. Applying Buchstab's identity twice, we have

$$
\begin{aligned}
& \text { (19) } \Psi_{1}=\sum_{v^{\frac{7.8}{25}}<p \leq v^{\frac{11.5}{25}}} \sum_{\frac{v^{\frac{13}{25}}}{p}<q<v^{\frac{5.2}{25}}} S\left(\mathcal{A}_{p q}, v^{\frac{1}{25}}\right) \\
& v^{\frac{26}{175}}<q<\frac{v^{\frac{9.55}{25}}}{p^{\frac{1}{2}}} \\
& -\sum_{v^{\frac{7.8}{25}}<p \leq v^{\frac{11.5}{25}}} \sum_{\substack{\frac{13}{25} \\
p}<<v^{\frac{5.2}{25}}} \sum_{v^{\frac{1}{25}<r<q}} S\left(\mathcal{A}_{p q r}, v^{\frac{1}{25}}\right) \\
& v^{\frac{26}{175}}<q<\frac{v^{\frac{9.55}{25}}}{p^{\frac{1}{2}}}
\end{aligned}
$$

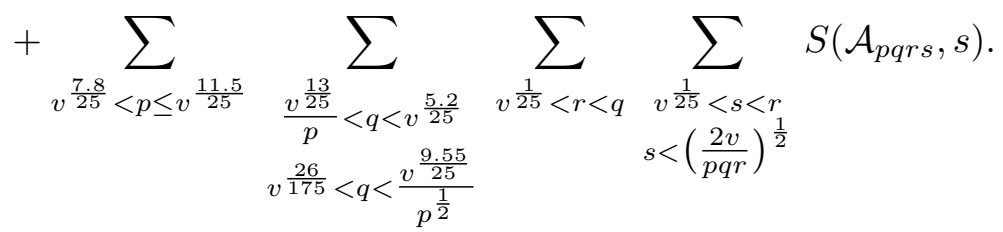

The first sum has an asymptotic formula. For the second sum

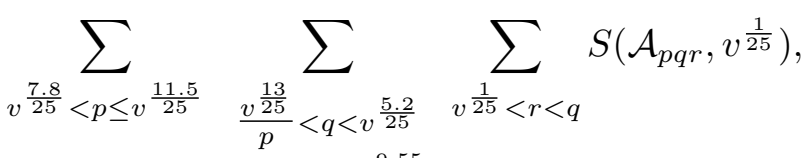

$$
\begin{aligned}
& v^{\frac{26}{175}}<q<\frac{v^{\frac{9.55}{25}}}{p^{\frac{1}{2}}}
\end{aligned}
$$


if $p r \leq v^{\frac{13}{25}}$, then we have an asymptotic formula. If $r \leq q^{-\frac{3}{4}} v^{\frac{6.5}{25}}$, then writing $m=p, n=q$ and $d=r$, we also have an asymptotic formula. Hence, we assume that $p r>v^{\frac{13}{25}}, r>q^{-\frac{3}{4}} v^{\frac{6.5}{25}}$ and have to deal with the sum

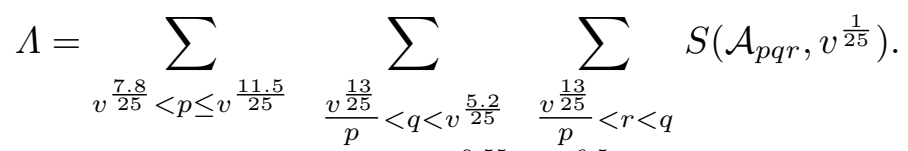

$$
\begin{aligned}
& v^{\frac{26}{175}}<q<\frac{v^{\frac{9.55}{25}}}{p^{\frac{1}{2}}} \quad \frac{v^{\frac{6.5}{25}}}{q^{\frac{3}{4}}}<r
\end{aligned}
$$

Now we use the discussion in [6]. Applying Buchstab's identity to the $p$-summation in $\Lambda$, we have

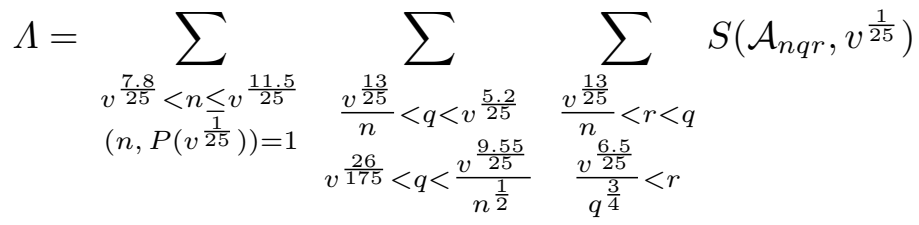

$$
\begin{aligned}
& -\sum_{\substack{v^{\frac{7.8}{25}}<z \nu \leq v^{\frac{11.5}{25}} \\
v^{\frac{1}{25}} \leq z<\nu \\
(\nu, P(z))=1}} \sum_{\substack{v^{\frac{13}{25}} z \nu \\
z \nu}} \sum_{\substack{\frac{26}{175}<v^{\frac{5.2}{25}} \\
\frac{v^{\frac{13}{25}}}{z \nu^{2}}<r<q \\
(z \nu)^{\frac{9.55}{25}}}} S\left(\mathcal{A}_{z \nu q r}, v^{\frac{1}{25}}\right),
\end{aligned}
$$

where $n, \nu$ denote integers and $z$ denotes a prime number.

We write

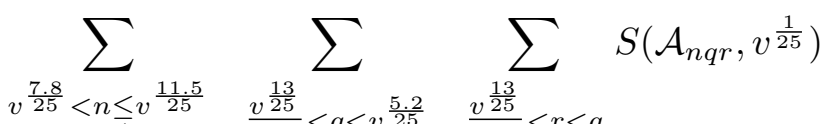

$$
\begin{aligned}
& \left(n, P\left(v \frac{1}{25}\right)\right)=1 \quad \frac{1}{n}<q<v \frac{525}{n} \quad \frac{125}{n}<r<q \\
& v^{\frac{26}{175}}<q<\frac{v^{\frac{9.55}{25}}}{n^{\frac{1}{2}}} \quad \frac{v^{\frac{6.5}{25}}}{q^{\frac{3}{4}}}<r \\
& =\#\left\{n q r l \in \mathcal{A}: v^{\frac{7.8}{25}}<n \leq v^{\frac{11.5}{25}},\left(n, P\left(v^{\frac{1}{25}}\right)\right)=1, v^{\frac{13}{25}} / n<q<v^{\frac{5.2}{25}}\right. \text {, } \\
& \left.v^{\frac{26}{175}}<q<v^{\frac{9.55}{25}} / n^{\frac{1}{2}}, v^{\frac{13}{25}} / n<r<q, v^{\frac{6.5}{25}} / q^{\frac{3}{4}}<r,\left(l, P\left(v^{\frac{1}{25}}\right)\right)=1\right\} .
\end{aligned}
$$

Note that $v^{\frac{13}{25}}<n r \Rightarrow q l \ll v^{\frac{12}{25}}$ and that $r<q<v^{\frac{6.5}{25}}$. By the discussion in Lemma 9 of [8] with the application of Lemma 2, we can get an asymptotic formula. The idea of transferring the application of the sieve method from the $l$-summation to the $n$-summation comes from [6].

Now we shall deal with the deficiencies of the following two sums:

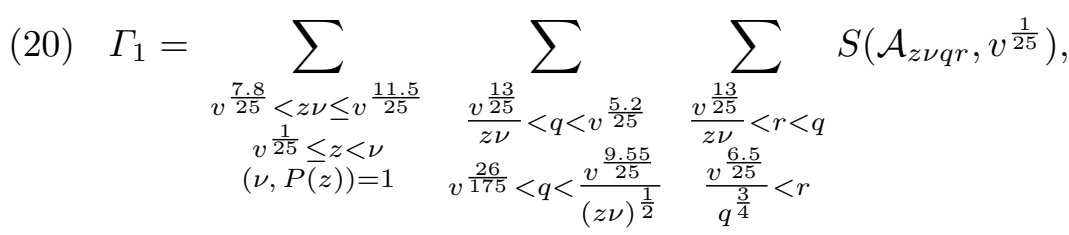




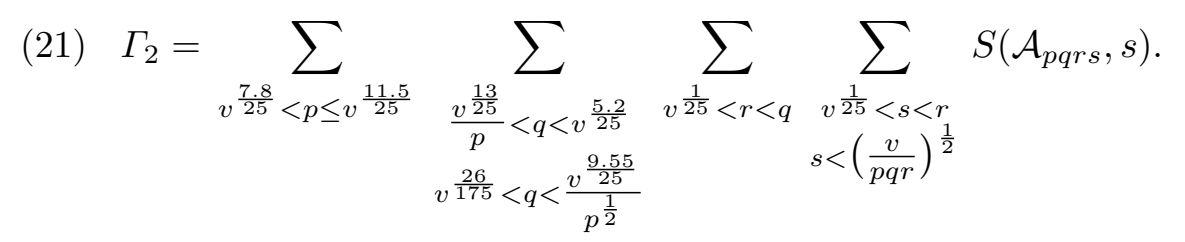

By Lemma 5 , the deficiency of $\Gamma_{1}$ is

$$
\begin{aligned}
& \int_{\frac{7.8}{25}}^{\frac{11.5}{25}} d t \int_{\max \left(\frac{13}{25}-t, \frac{26}{175}\right)}^{\min \left(\frac{5.2}{25}, \frac{9.55}{25}-\frac{t}{2}\right)} \frac{d u}{u} \int_{\frac{1}{25}}^{\frac{t}{2}} w\left(\frac{t-z}{z}\right) \frac{d z}{z^{2}} \\
& \times \int_{\max \left(\frac{13}{25}-t, \frac{6.5}{25}-\frac{3}{4} u\right)}^{u} 25 w(25(1-t-u-r)) \frac{d r}{r}
\end{aligned}
$$

and that of $\Gamma_{2}$ is

$$
\begin{aligned}
\int_{\frac{7.8}{25}}^{\frac{11.5}{25}} \frac{d t}{t} \int_{\max \left(\frac{13}{25}-t, \frac{26}{175}\right)}^{\min \left(\frac{5.2}{25}, \frac{9.55}{25}-\frac{t}{2}\right)} \frac{d u}{u} \int_{\frac{1}{25}}^{u} \frac{d r}{r} \\
\times \int_{\frac{1}{25}}^{\min \left(r, \frac{1}{2}(1-t-u-r)\right)} w\left(\frac{1-t-u-r-s}{s}\right) \frac{d s}{s^{2}} .
\end{aligned}
$$

We refer to [6] and [10]. Firstly we consider the deficiency of $\Gamma_{1}$ in some cases.

1. $r \nu>v^{\frac{13}{25}}$. The corresponding deficiency is

$$
\begin{aligned}
\int_{\frac{8.9}{25}}^{\frac{11.5}{25}} d t \int_{\max \left(\frac{14}{25}-t, \frac{26}{175}\right)}^{\frac{9.55}{25}-\frac{t}{2}} \frac{d u}{u} \int_{\frac{1}{25}}^{t+u-\frac{13}{25}} w\left(\frac{t-z}{z}\right) \frac{d z}{z^{2}} \\
\times \int_{\max \left(\frac{13}{25}-t+z, \frac{6.5}{25}-\frac{3}{4} u\right)}^{u} 25 w(25(1-t-u-r)) \frac{d r}{r} .
\end{aligned}
$$

We use Buchstab's identity

$$
S\left(\mathcal{A}_{z \nu q r}, v^{\frac{1}{25}}\right)=S\left(\mathcal{A}_{z \nu q r}, r\right)+\sum_{\substack{\frac{1}{25} \leq s<r \\ s<\left(\frac{2 v}{z \nu q r}\right)^{\frac{1}{2}}}} S\left(\mathcal{A}_{z \nu q r s}, s\right)
$$

to produce two sums

$$
\Lambda_{1}=\sum_{z} \sum_{\nu} \sum_{q} \sum_{r} S\left(\mathcal{A}_{z \nu q r}, r\right),
$$




$$
\Lambda_{2}=\sum_{z} \sum_{\nu} \sum_{q} \sum_{r} \sum_{\substack{\frac{1}{25} \leq s<r \\ s<\left(\frac{2 v}{z \nu q r}\right)^{\frac{1}{2}}}} S\left(\mathcal{A}_{z \nu q r s}, s\right) .
$$

1) The deficiency of $\Lambda_{1}$ is

$$
\begin{aligned}
\int_{\frac{8.9}{25}}^{\frac{11.5}{25}} d t \int_{\max \left(\frac{14}{25}-t, \frac{26}{175}\right)}^{\frac{9.55}{25}-\frac{t}{2}} \frac{d u}{u} \int_{\frac{1}{25}}^{t+u-\frac{13}{25}} w\left(\frac{t-z}{z}\right) \frac{d z}{z^{2}} \\
\times \int_{\max \left(\frac{13}{25}-t+z, \frac{6.5}{25}-\frac{3}{4} u\right)}^{u} w\left(\frac{1-t-u-r}{r}\right) \frac{d r}{r^{2}} .
\end{aligned}
$$

Applying process II to the $\nu$-summation, we have to consider the prime term and the almost-prime term. For convenience, we call them the $z$-prime term and the $z$-almost-prime term respectively.

i) The deficiency of the $z$-prime term is

$$
\begin{aligned}
\int_{\frac{8.9}{25}}^{\frac{11.5}{25}} d t \int_{\max \left(\frac{14}{25}-t, \frac{26}{175}\right)}^{\frac{9.55}{25}-\frac{t}{2}} \frac{d u}{u} \int_{\frac{1}{25}}^{t+u-\frac{13}{25}} \frac{d z}{z(t-z)} \\
\times \int_{\max \left(\frac{13}{25}-t+z, \frac{6.5}{25}-\frac{3}{4} u\right)}^{u} g\left(\frac{1-t-u-r}{r}\right) \frac{d r}{r^{2}} \leq 0.002064 .
\end{aligned}
$$

ii) The deficiency of the $z$-almost-prime term. Now $\nu$ is replaced by $e \beta$, where $z<e<\beta,(\beta, P(e))=1, e$ is a prime number and $\beta$ is an integer. The corresponding deficiency is

$$
\begin{aligned}
& \int_{\frac{8.9}{25}}^{\frac{11.5}{25}} d t \int_{\max \left(\frac{14}{25}-t, \frac{26}{175}\right)}^{\frac{9.55}{25}-\frac{t}{2}} \frac{d u}{u} \int_{\frac{1}{25}}^{t+u-\frac{13}{25}} \frac{d z}{z} \\
& \times \int_{z}^{\frac{1}{2}(t-z)} w\left(\frac{t-z-e}{e}\right) \frac{d e}{e^{2}} \int_{\max \left(\frac{13}{25}-t+z, \frac{6.5}{25}-\frac{3}{4} u\right)}^{u} w\left(\frac{1-t-u-r}{r}\right) \frac{d r}{r^{2}} .
\end{aligned}
$$

a) $q r e>v^{\frac{13}{25}}(0.000029)$. b) qre $<v^{\frac{12}{25}}$, qrze $>v^{\frac{13}{25}}(0.000229)$. c) $\left.\left.q r z e<v^{\frac{12}{25}}: \alpha\right) r \beta>v^{\frac{13}{25}}(0.000006) ; \beta\right) r \beta<v^{\frac{12}{25}}, r z \beta>v^{\frac{13}{25}}(0.000644)$; ᄀ) $r z \beta<v^{\frac{12}{25}}(0.000463)$.

Therefore the total deficiency of $\Lambda_{1}$ is 0.003435 . 
2) The deficiency of $\Lambda_{2}$ is

$$
\begin{aligned}
\int_{\frac{8.9}{25}}^{\frac{11.5}{25}} d t & \int_{\max \left(\frac{14}{25}-t, \frac{26}{175}\right)}^{\frac{9.55}{25}-\frac{t}{2}} \frac{d u}{u} \int_{\frac{1}{25}}^{t+u-\frac{13}{25}} w\left(\frac{t-z}{z}\right) \frac{d z}{z^{2}} \\
& \times \int_{\max \left(\frac{13}{25}-t+z, \frac{6.5}{25}-\frac{3}{4} u\right)}^{u} \frac{d r}{r} \int_{\frac{1}{25}}^{\min \left(r, \frac{1}{2}(1-t-u-r)\right)} w\left(\frac{1-t-u-r-s}{s}\right) \frac{d s}{s^{2}} .
\end{aligned}
$$

i) $s \nu>v^{\frac{13}{25}}(0.000102)$. ii) $s \nu<v^{\frac{12}{25}}, s z \nu>v^{\frac{13}{25}}(0.001319)$.

iii) $s z \nu<v^{\frac{12}{25}}$. The corresponding deficiency is

$$
\begin{aligned}
& \int_{\frac{8.9}{25}}^{\frac{11}{25}} d t \int_{\max \left(\frac{14}{25}-t, \frac{26}{175}\right)}^{\frac{9.55}{25}-\frac{t}{2}} \frac{d u}{u} \int_{\frac{1}{25}}^{t+u-\frac{13}{25}} w\left(\frac{t-z}{z}\right) \frac{d z}{z^{2}} \\
& \times \int_{\max \left(\frac{13}{25}-t+z, \frac{6.5}{25}-\frac{3}{4} u\right)}^{u} \frac{d r}{r} \int_{\frac{1}{25}}^{\min \left(\frac{1}{2}(1-t-u-r), \frac{12}{25}-t\right)} w\left(\frac{1-t-u-r-s}{s}\right) \frac{d s}{s^{2}} .
\end{aligned}
$$

a) The deficiency of the $z$-prime term is 0.001807 .

b) The deficiency of the $z$-almost-prime term is

$$
\begin{aligned}
& \int_{\frac{8.9}{25}}^{\frac{11}{25}} d t \int_{\max \left(\frac{14}{25}-t, \frac{26}{175}\right)}^{\frac{9.55}{25}-\frac{t}{2}} \frac{d u}{u} \int_{\frac{1}{25}}^{t+u-\frac{13}{25}} \frac{d z}{z} \int_{z}^{\frac{1}{2}(t-z)} w\left(\frac{t-z-e}{e}\right) \frac{d e}{e^{2}} \\
& \times \quad \int_{\max \left(\frac{13}{25}-t+z, \frac{6.5}{25}-\frac{3}{4} u\right)}^{u} \frac{d r}{r} \int_{\frac{1}{25}}^{\min \left(\frac{1}{2}(1-t-u-r), \frac{12}{25}-t\right)} w\left(\frac{1-t-u-r-s}{s}\right) \frac{d s}{s^{2}} .
\end{aligned}
$$

a) $\left.\left.r \beta>v^{\frac{13}{25}}(0.000006) . \beta\right) r \beta<v^{\frac{12}{25}}, r z \beta>v^{\frac{13}{25}}(0.000471) . \gamma\right) r z \beta<$ $\left.v^{\frac{12}{25}}, r s z \beta>v^{\frac{13}{25}}(0.000396) . \delta\right) r s z \beta<v^{\frac{12}{25}}, q s z \beta>v^{\frac{13}{25}}(0.000006)$. ג) $q s z \beta<v^{\frac{12}{25}}(0.000611)$.

Therefore the total deficiency of $\Lambda_{2}$ is 0.004718 and that in 1 . is 0.008153 .

2. $r \nu<v^{\frac{12}{25}}, q \nu>v^{\frac{13}{25}}$. The corresponding deficiency is

$$
\begin{aligned}
\int_{\frac{8.9}{25}}^{\frac{73.7}{175}} d t \int_{\max \left(\frac{14}{25}-t, \frac{30}{175}\right)}^{\frac{9.55}{25}-\frac{t}{2}} \frac{d u}{u} & \int_{\max \left(\frac{1}{25}, t-\frac{3}{4} u-\frac{5.5}{25}\right)}^{t+u-\frac{13}{25}} w\left(\frac{t-z}{z}\right) \frac{d z}{z^{2}} \\
& \times \int_{\max \left(\frac{13}{25}-t, \frac{6.5}{25}-\frac{3}{4} u\right)}^{\frac{12}{25}-t+z} 25 w(25(1-t-u-r)) \frac{d r}{r} .
\end{aligned}
$$

Note that $z<q^{-\frac{3}{4}} v^{\frac{6.5}{25}}$. Let $m=r \nu, n=q$ and $d=z$. Then we have an asymptotic formula. Hence, the deficiency is 0 . 
3. $q \nu<v^{\frac{12}{25}}$. The corresponding deficiency is

$$
\begin{aligned}
\int_{\frac{7.8}{25}}^{\frac{11.5}{25}} d t \int_{\max \left(\frac{13}{25}-t, \frac{26}{175}\right)}^{\min \left(\frac{5.2}{25}, \frac{9.55}{25}-\frac{t}{2}\right)} \frac{d u}{u} \int_{t+u-\frac{12}{25}}^{\frac{t}{2}} w\left(\frac{t-z}{z}\right) \frac{d z}{z^{2}} \\
\times \int_{\max \left(\frac{13}{25}-t, \frac{6.5}{25}-\frac{3}{4} u\right)}^{u} 25 w(25(1-t-u-r)) \frac{d r}{r} .
\end{aligned}
$$

1) $q r z>v^{\frac{13}{25}}$.

i) The deficiency of $\Lambda_{1}$ is

$$
\begin{aligned}
\int_{\frac{7.8}{25}}^{\frac{11.5}{25}} d t \int_{\max \left(\frac{13}{25}-t, \frac{26}{175}, \frac{6.5}{25}-\frac{t}{4}\right)}^{\min \left(\frac{5.2}{25}, \frac{9.55}{25}-\frac{t}{2}\right)} \frac{d u}{u} \int_{\frac{13}{25}-2 u}^{\frac{t}{2}} g\left(\frac{t-z}{z}\right) \frac{d z}{z^{2}} \\
\times \int_{\max \left(\frac{13}{25}-t, \frac{6.5}{25}-\frac{3}{4} u, \frac{13}{25}-u-z\right)}^{u} g\left(\frac{1-t-u-r}{r}\right) \frac{d r}{r^{2}}
\end{aligned}
$$$$
\leq 0.004218 \text {. }
$$

ii) The deficiency of $\Lambda_{2}$ is

$$
\begin{aligned}
& \int_{\frac{7.8}{25}}^{\frac{11.5}{25}} d t \int_{\max \left(\frac{13}{25}-t, \frac{26}{175}, \frac{6.5}{25}-\frac{t}{4}\right)}^{\min \left(\frac{5.2}{25}, \frac{9.55}{25}-\frac{t}{2}\right)} \frac{d u}{u} \int_{\frac{13}{25}-2 u}^{\frac{t}{2}} w\left(\frac{t-z}{z}\right) \frac{d z}{z^{2}} \\
& \times \quad \int_{\max \left(\frac{13}{25}-t, \frac{6.5}{25}-\frac{3}{4} u, \frac{13}{25}-u-z\right)}^{u} \frac{d r}{r} \int_{\frac{1}{25}}^{\min \left(r, \frac{1}{2}(1-t-u-r)\right)} w\left(\frac{1-t-u-r-s}{s}\right) \frac{d s}{s^{2}} .
\end{aligned}
$$

a) $s z \nu>v^{\frac{13}{25}}(0.000236)$.

b) $s z \nu<v^{\frac{12}{25}}$.

a) qrs $>v^{\frac{13}{25}}(0.000146)$. $\left.\left.\beta\right) q r s<v^{\frac{12}{25}}: \beta_{1}\right) q s \nu>v^{\frac{13}{25}}(0.000006)$; $\left.\beta_{2}\right) q s \nu<v^{\frac{12}{25}}(0.007355)$.

Therefore the total deficiency in 1) is 0.011961 .

2) $q r z<v^{\frac{12}{25}}$. If $\nu \leq v^{\frac{6.5}{25}}$, writing $m=q r z$ and $n=\nu$, we have an asymptotic formula. Hence, we assume $\nu>v^{\frac{6.5}{25}}$. The corresponding deficiency is

$$
\begin{aligned}
\int_{\frac{7.8}{25}}^{\frac{11.5}{25}} d t \int_{\max \left(\frac{13}{25}-t, \frac{26}{175}\right)}^{\min \left(\frac{5.2}{25}, \frac{9.55}{25}-\frac{t}{2}\right)} \frac{d u}{u} \int_{t+u-\frac{12}{25}}^{\min \left(t-\frac{6.5}{25}, \frac{5.5}{25}-\frac{u}{4}\right)} w\left(\frac{t-z}{z}\right) \frac{d z}{z^{2}} \\
\times \int_{\max \left(\frac{13}{25}-t, \frac{6.5}{25}-\frac{3}{4} u\right)}^{\min \left(u, \frac{12}{25}-u-z\right)} 25 w(25(1-t-u-r)) \frac{d r}{r} .
\end{aligned}
$$


i) $r \leq v^{\frac{26}{175}}$. The corresponding deficiency is

$$
\begin{aligned}
\int_{\frac{65}{175}}^{\frac{11.5}{25}} d t \int_{\frac{26}{175}}^{\min \left(\frac{5.2}{25}, \frac{9.55}{25}-\frac{t}{2}\right)} \frac{d u}{u} \int_{t+u-\frac{12}{25}}^{\min \left(t-\frac{6.5}{25}, \frac{5.5}{25}-\frac{u}{4}\right)} w\left(\frac{t-z}{z}\right) \frac{d z}{z^{2}} \\
\times \int_{\max \left(\frac{13}{25}-t, \frac{6.5}{25}-\frac{3}{4} u\right)}^{\min \left(\frac{12}{25}-u-z, \frac{26}{175}\right)} 25 w(25(1-t-u-r)) \frac{d r}{r} .
\end{aligned}
$$

Note that $v^{\frac{2.6}{25}}<r$. If $z<r^{-\frac{4}{3}} v^{\frac{26}{75}}\left(r<z^{-\frac{3}{4}} v^{\frac{6.5}{25}}\right)$, writing $m=q \nu, n=r$ and $d=z$, we have an asymptotic formula. Hence, we assume $r \geq z^{-\frac{3}{4}} v^{\frac{6.5}{25}}$. The deficiency is

$$
\begin{aligned}
\int_{\frac{71.5}{175}}^{\frac{11.5}{25}} d t \int_{\frac{26}{175}}^{\frac{9.55}{25}-\frac{t}{2}} \frac{d u}{u} & \int_{\frac{26}{175}}^{\min \left(t-\frac{6.5}{25}, \frac{5.5}{25}-\frac{u}{4}\right)} g\left(\frac{t-z}{z}\right) \frac{d z}{z^{2}} \\
& \times \int_{\max \left(\frac{13}{25}-t, \frac{6.5}{25}-\frac{3}{4} u, \frac{6.5}{25}-\frac{3}{4} z\right)}^{\min \left(\frac{12}{25}-u-z, \frac{26}{175}\right)} 25 g(25(1-t-u-r)) \frac{d r}{r}
\end{aligned}
$$$$
\leq 0.000754 \text {. }
$$

ii) $v^{\frac{26}{175}}<r\left(<v^{\frac{5.2}{25}}\right)$. The corresponding deficiency is

$$
\begin{aligned}
\int_{\frac{7.8}{25}}^{\frac{11.5}{25}} d t \int_{\max \left(\frac{13}{25}-t, \frac{26}{175}\right)}^{\min \left(\frac{5.2}{25}, \frac{9.55}{25}-\frac{t}{2}\right)} \frac{d u}{u} \int_{t+u-\frac{12}{25}}^{\min \left(t-\frac{6.5}{25}, \frac{58}{175}-u\right)} w\left(\frac{t-z}{z}\right) \frac{d z}{z^{2}} \\
\times \int_{\max \left(\frac{13}{25}-t, \frac{26}{175}\right)}^{\min \left(u, \frac{12}{25}-u-z\right)} 25 w(25(1-t-u-r)) \frac{d r}{r} .
\end{aligned}
$$

If $z<r^{-\frac{3}{4}} v^{\frac{6.5}{25}}\left(r<z^{-\frac{4}{3}} v^{\frac{26}{75}}\right)$, writing $m=q \nu, n=r$ and $d=z$, we have an asymptotic formula. Hence, we assume $r \geq z^{-\frac{4}{3}} v^{\frac{26}{75}}$. The deficiency is

$$
\begin{aligned}
& \int_{\frac{7.8}{25}}^{\frac{11.5}{25}} d t \int_{\max \left(\frac{13}{25}-t, \frac{26}{175}\right)}^{\min \left(\frac{5.2}{25}, \frac{9.55}{25}-\frac{t}{2}\right)} \frac{d u}{u} \int_{t+u-\frac{12}{25}}^{\min \left(t-\frac{6.5}{25}, \frac{58}{175}-u\right)} g\left(\frac{t-z}{z}\right) \frac{d z}{z^{2}} \\
& \times \quad \int_{\max \left(\max \left(\frac{13}{25}-t, \frac{26}{175}, \frac{26}{75}-\frac{4}{3} z\right), \min \left(u, \frac{12}{25}-u-z\right)\right)} 25 g(25(1-t-u-r)) \frac{d r}{r}
\end{aligned}
$$$$
\leq 0.001733 \text {. }
$$

Thus the deficiency in 2) is 0.002487 and that in 3 . is 0.014448 . Therefore the deficiency of $\Gamma_{1}$ is 0.022601 . 
Next we consider the deficiency of $\Gamma_{2}$.

1. $p s>v^{\frac{13}{25}}$. The deficiency is

$$
\begin{aligned}
\int_{\frac{9}{25}}^{\frac{11.5}{25}} \frac{d t}{t} \int_{\max \left(\frac{13}{25}-t, \frac{26}{175}\right)}^{\min \left(\frac{9.55}{25}-\frac{t}{2}, 2 t-\frac{14}{25}\right)} & \frac{d u}{u} \int_{\frac{13}{25}-t}^{\min \left(u, t-u-\frac{1}{25}\right)} \frac{d r}{r} \\
& \times \int_{\frac{13}{25}-t}^{\min \left(r, \frac{1}{2}(1-t-u-r)\right)} g\left(\frac{1-t-u-r-s}{s}\right) \frac{d s}{s^{2}}
\end{aligned}
$$$$
\leq 0.013694 \text {. }
$$

2. $p s<v^{\frac{12}{25}}, p r>v^{\frac{13}{25}}$. The corresponding deficiency is

$$
\begin{aligned}
\int_{\frac{7.8}{25}}^{\frac{11}{25}} \frac{d t}{t} \int_{\max \left(\frac{13}{25}-t, \frac{26}{175}\right)}^{\min \left(\frac{5.2}{25}, \frac{9.55}{25}-\frac{t}{2}\right)} \frac{d u}{u} \int_{\frac{13}{25}-t}^{u} \frac{d r}{r} \\
\times \int_{\frac{1}{25}}^{\min \left(\frac{1}{2}(1-t-u-r), \frac{12}{25}-t\right)} w\left(\frac{1-t-u-r-s}{s}\right) \frac{d s}{s^{2}} .
\end{aligned}
$$

1) $q r s>v^{\frac{13}{25}}(0.000236)$.

2) $q r s<v^{\frac{12}{25}}$.

i) The deficiency of the prime term is 0.036198 .

ii) The deficiency of the almost-prime term is

$$
\begin{aligned}
\int_{\frac{7.8}{25}}^{\frac{11}{25}} \frac{d t}{t} \int_{\max \left(\frac{13}{25}-t, \frac{26}{175}\right)}^{\min \left(\frac{5.2}{25}, \frac{9.55}{25}-\frac{t}{2}\right)} \frac{d u}{u} \int_{\frac{13}{25}-t}^{u} \frac{d r}{r} \int_{\frac{1}{25}}^{\min \left(\frac{12}{25}-t, \frac{12}{25}-u-r, \frac{1}{3}(1-t-u-r)\right)} \frac{d s}{s} \\
\times \int_{s}^{\frac{1}{2}(1-t-u-r-s)} w\left(\frac{1-t-u-r-s-k}{k}\right) \frac{d k}{k^{2}} .
\end{aligned}
$$

a) $k p>v^{\frac{13}{25}}(0.002569)$. b) $\left.k p<v^{\frac{12}{25}}, k p s>v^{\frac{13}{25}}: \alpha\right) k q r s>v^{\frac{13}{25}}$ $(0.000774)$; $\beta) k q r s<v^{\frac{12}{25}}(0.004275)$. c) kps $\left.<v^{\frac{12}{25}}: \alpha\right) k q r s>v^{\frac{13}{25}}(0.000402)$;

B) $k q r s<v^{\frac{12}{25}}(0.003268)$.

Therefore the total deficiency in 2. is 0.047722 .

3. $p r<v^{\frac{12}{25}}$, prs $>v^{\frac{13}{25}}$. The corresponding deficiency is

$$
\int_{\frac{7.8}{25}}^{\frac{11}{25}} \frac{d t}{t} \int_{\max \left(\frac{13}{25}-t, \frac{26}{175}\right)}^{\min \left(\frac{5.2}{25}, \frac{9.55}{25}-\frac{t}{2}\right)} \frac{d u}{u} \int_{\frac{1}{2}\left(\frac{13}{25}-t\right)}^{\frac{12}{25}-t} \frac{d r}{r} \int_{\frac{13}{25}-t-r}^{\min \left(r, \frac{1}{2}(1-t-u-r)\right)} w\left(\frac{1-t-u-r-s}{s}\right) \frac{d s}{s^{2}} .
$$


1) $s<q^{-\frac{3}{8}} v^{\frac{6.5}{50}}$. Let $m=p r, n=q$ and $d=s t$. Applying process I with Lemma 3 , we have to deal with a sum whose deficiency is

$$
\begin{aligned}
\int_{\frac{7.8}{25}}^{\frac{11}{25}} \frac{d t}{t} & \int_{\max \left(\frac{13}{25}-t, \frac{26}{175}\right)}^{\min \left(\frac{5.2}{25}, \frac{9.55}{25}-\frac{t}{2}\right)} \frac{d u}{u} \int_{\max \left(\frac{1}{2}\left(\frac{13}{25}-t\right), \frac{19.5}{50}-t+\frac{3}{8} u\right)}^{\frac{12}{25}-t} \frac{d r}{r} \\
& \times \int_{\frac{13}{25}-t-r}^{\min \left(r, \frac{6.5}{50}-\frac{3}{8} u\right)} \frac{d s}{s} \int_{\frac{1}{25}}^{s} \frac{d y}{y} \int_{\frac{1}{25}}^{y} w\left(\frac{1-t-u-r-s-y-z}{z}\right) \frac{d z}{z^{2}} .
\end{aligned}
$$

i) $p r w>v^{\frac{13}{25}}(0.000643)$. ii) $p r w<v^{\frac{12}{25}}(0)$.

Hence, the total deficiency in 1 ) is 0.000643 .

2) $s>q^{-\frac{3}{8}} v^{\frac{6.5}{50}}$.

i) The deficiency of the prime term is 0.021656 .

ii) The deficiency of the almost-prime term is

$$
\begin{aligned}
& \int_{\frac{7.8}{25}}^{\frac{11}{25}} \frac{d t}{t} \int_{\max \left(\frac{13}{25}-t, \frac{26}{175}\right)}^{\min \left(\frac{5.2}{25}, \frac{9.55}{25}-\frac{t}{2}\right)} \frac{d u}{u} \int_{\frac{1}{2}\left(\frac{13}{25}-t\right)}^{\frac{12}{25}-t} \frac{d r}{r} \\
& \times \int_{s}^{\max \left(\max \left(\frac{13}{25}-t-r, \frac{6.5}{50}-\frac{3}{8} u\right), \min \left(r, \frac{1}{3}(1-t-u-r)\right)\right)} \frac{d s}{s} \\
& \times \int_{\max \left(\frac{13}{25}-t-r, \frac{6.5}{50}-\frac{3}{8} u\right)} \int^{\frac{1}{2}(1-t-u-r-s)} w\left(\frac{1-t-u-r-s-k}{k}\right) \frac{d k}{k^{2}} .
\end{aligned}
$$

a) $k p>v^{\frac{13}{25}}(0.000349)$. b) $\left.k p<v^{\frac{12}{25}}, k p s>v^{\frac{13}{25}}: \alpha\right) k q r s>v^{\frac{13}{25}}$ (0.001240); $\beta) k q r s<v^{\frac{12}{25}}(0.004339)$. c) kps $<v^{\frac{12}{25}}(0.000786)$.

Therefore the total deficiency in 2) is 0.028370 and that in 3 . is 0.029013 .

4. prs $<v^{\frac{12}{25}}$. The corresponding deficiency is

$$
\int_{\frac{7.8}{25}}^{\frac{10}{25}} \frac{d t}{t} \int_{\max \left(\frac{13}{25}-t, \frac{26}{175}\right)}^{\min \left(\frac{5.2}{25}, \frac{9.55}{25}-\frac{t}{2}\right)} \frac{d u}{u} \int_{\frac{1}{25}}^{\frac{11}{25}-t} \frac{d r}{r} \int_{\frac{1}{25}}^{\min \left(r, \frac{12}{25}-t-r\right)} w\left(\frac{1-t-u-r-s}{s}\right) \frac{d s}{s^{2}} .
$$

Let $m=p r s, n=q$ and $d=t$. Note that $t<s<\min \left(r, v^{\frac{12}{25}} / p r\right) \leq$ $\left(v^{\frac{12}{25}} / p\right)^{\frac{1}{2}}<v^{\frac{6.5}{25}} q^{-\frac{3}{4}}$. Applying process I with Lemma 3, we have to deal with a sum whose deficiency is 


$$
\begin{aligned}
\int_{\frac{7.8}{25}}^{\frac{10}{25}} \frac{d t}{t} \int_{\max \left(\frac{13}{25}-t, \frac{26}{175}\right)}^{\min \left(\frac{5.2}{25}, \frac{9.55}{25}-\frac{t}{2}\right)} \frac{d u}{u} \int_{\frac{1}{25}}^{\frac{11}{25}-t} \frac{d r}{r} \int_{\frac{1}{25}}^{\min \left(r, \frac{12}{25}-t-r\right)} \frac{d s}{s} \int_{\frac{1}{25}}^{s} \frac{d y}{y} \\
\times \int_{\frac{1}{25}}^{\min \left(y, \frac{1}{2}(1-t-u-r-s-y)\right)} w\left(\frac{1-t-u-r-s-y-z}{z}\right) \frac{d z}{z^{2}}
\end{aligned}
$$

1) $\left.p r s w>v^{\frac{13}{25}}(0.000373) .2\right)$ prsw $<v^{\frac{12}{25}}(0.000063)$.

Thus the total deficiency in 4 . is 0.000436 . Hence, the deficiency of $\Gamma_{2}$ is 0.090865 . The deficiency of $\Psi_{1}$ is 0.113466 and that of $\Psi_{2}$ is 0.157504 . Therefore the total deficiency in III is 0.270970 .

IV. $q>v^{\frac{5.2}{25}}$. The deficiency is

$$
\int_{\frac{6.5}{25}}^{\frac{12}{25}} \frac{d t}{t} \int_{\max \left(\frac{13}{25}-t, \frac{5.2}{25}\right)}^{\min \left(t, \frac{1}{2}(1-t)\right)} g\left(\frac{1-t-u}{u}\right) \frac{d u}{u^{2}} \leq 0.529493 .
$$

Therefore the deficiency of $\Omega_{2}$ is 0.891757 .

6. Proof of the Theorem. The above discussion yields

$$
\begin{aligned}
\Phi \geq & \frac{X}{v} \cdot\left(S\left(\mathcal{B}, v^{\frac{1}{25}}\right)-\sum_{v^{\frac{1}{25}}<p \leq v^{\frac{12}{25}}} S\left(\mathcal{B}_{p}, p\right)-\sum_{v^{\frac{12}{25}<p \leq(2 v)^{\frac{1}{2}}}} S\left(\mathcal{B}_{p}, p\right)\right) \\
& -0.096767 \cdot \frac{X}{\log v}-0.891757 \cdot \frac{X}{\log v} \\
= & \frac{X}{v} \cdot S\left(\mathcal{B},(2 v)^{\frac{1}{2}}\right)-0.988524 \cdot \frac{X}{\log v} \\
= & (1+O(\varepsilon)) \cdot \frac{X}{\log v}-0.988524 \cdot \frac{X}{\log v} \\
\geq & 0.011475 \cdot \frac{X}{\log v} .
\end{aligned}
$$

Thus (3) holds and so the proof of the Theorem is complete.

Acknowledgements. We would like to thank the referee for his helpful comments and news on the recent progress in this problem. In an unpublished manuscript, Harman got the exponent $\varphi=\frac{19}{20}$. In his doctoral thesis (Oxford, 1998), J. K. Haugland got $\varphi=\frac{24}{25}$.

\section{References}

[1] A. Balog, Numbers with a large prime factor, Studia Sci. Math. Hungar. 15 (1980), 139-146. 
[2] A. Balog, Numbers with a large prime factor II, in: Topics in Classical Number Theory, Colloq. Math. Soc. János Bolyai 34, Elsevier, North-Holland, Amsterdam, 1984, 49-67.

[3] A. Balog, G. Harman and J. Pintz, Numbers with a large prime factor IV, J. London Math. Soc. (2) 28 (1983), 218-226.

[4] J.-M. Deshouillers and H. Iwaniec, Power mean-values for Dirichlet's polynomials and the Riemann zeta-function, II, Acta Arith. 43 (1984), 305-312.

[5] G. Harman, On the distribution of $\alpha p$ modulo one, J. London Math. Soc. (2) 27 (1983), 9-18.

[6] -, On the distribution of $\alpha p$ modulo one II, Proc. London Math. Soc. (3) 72 (1996), 241-260.

[7] D. R. Heath-Brown, The largest prime factor of the integers in an interval, Sci. China Ser. A 26 (1996), 385-411 (in Chinese); Sci. China Ser. A 39 (1996), 449-476.

[8] D. R. Heath-Brown and C. Jia, The largest prime factor of the integers in an interval, II, J. Reine Angew. Math. 498 (1998), 35-59.

[9] C. Jia, On the distribution of $\alpha p$ modulo one, J. Number Theory 45 (1993), 241-253.

[10] - On the distribution of ap modulo one, II, preprint.

[11] M. Jutila, On numbers with a large prime factor, J. Indian Math. Soc. (N.S.) 37 (1973), 43-53.

Institute of Mathematics

Academia Sinica

Beijing, 100080, China

E-mail: jiach@math08.math.ac.cn
Department of Mathematics The University of Hong Kong Pokfulam Road, Hong Kong E-mail: matmcliu@hkucc.hku.hk

Received on 26.4.1999

and in revised form on 10.10.1999 